\title{
Multiresolution Decomposition Schemes Using the Parameterized Logarithmic Image Processing Model with Application to Image Fusion
}

\author{
Shahan C. Nercessian, ${ }^{1}$ Karen A. Panetta, ${ }^{1}$ and Sos S. Agaian ${ }^{2}$ \\ ${ }^{1}$ Department of Electrical and Computer Engineering, Tufts University, 161 College Avenue, Medford, MA 02155, USA \\ ${ }^{2}$ Department of Electrical and Computer Engineering, University of Texas at San Antonio, 6900 North Loop 1604 West, \\ San Antonio, TX 78249, USA
}

Correspondence should be addressed to Shahan C. Nercessian, shahan.nercessian@gmail.com

Received 23 June 2010; Revised 6 September 2010; Accepted 7 October 2010

Academic Editor: Dennis Deng

Copyright (C) 2011 Shahan C. Nercessian et al. This is an open access article distributed under the Creative Commons Attribution License, which permits unrestricted use, distribution, and reproduction in any medium, provided the original work is properly cited.

\begin{abstract}
New pixel- and region-based multiresolution image fusion algorithms are introduced in this paper using the Parameterized Logarithmic Image Processing (PLIP) model, a framework more suitable for processing images. A mathematical analysis shows that the Logarithmic Image Processing (LIP) model and standard mathematical operators are extreme cases of the PLIP model operators. Moreover, the PLIP model operators also have the ability to take on cases in between LIP and standard operators based on the visual requirements of the input images. PLIP-based multiresolution decomposition schemes are developed and thoroughly applied for image fusion as analysis and synthesis methods. The new decomposition schemes and fusion rules yield novel image fusion algorithms which are able to provide visually more pleasing fusion results. LIP-based multiresolution image fusion approaches are consequently formulated due to the generalized nature of the PLIP model. Computer simulations illustrate that the proposed image fusion algorithms using the Parameterized Logarithmic Laplacian Pyramid, Parameterized Logarithmic Discrete Wavelet Transform, and Parameterized Logarithmic Stationary Wavelet Transform outperform their respective traditional approaches by both qualitative and quantitative means. The algorithms were tested over a range of different image classes, including out-of-focus, medical, surveillance, and remote sensing images.
\end{abstract}

\section{Introduction}

Great advances in sensor technology have brought about the emerging field of image fusion. Image fusion is the combination of two or more source images which vary in resolution, instrument modality, or image capture technique into a single composite representation $[1,2]$. The goal of an image fusion algorithm is to integrate the redundant and complementary information obtained from the source images in order to form a new image which provides a better description of the scene for human or machine perception [3]. Thus, image fusion is essential for computer vision and robotics systems in which fusion results can be used to aid further processing steps for a given task. Image fusion techniques are practical and fruitful for many applications, including medical imaging, security, military, remote sensing, digital camera, and consumer use. In medical imaging, magnetic resonance imaging (MRI) and computed tomography (CT) images provide structural and anatomical information with high resolution. Positron emission tomography (PET) and single photon emission computed tomography (SPECT) images provide functional information with low resolution. Therefore, the fusion of MRI or CT images with PET or SPECT images can provide the needed structural, anatomical, and functional information for medical diagnosis, anomaly detection, and quantitative analysis [4]. Similarly, the combination of MRI and CT images can provide images containing both dense bone structure and normal or pathological soft tissue information [5]. In security applications, thermal/infrared images 
provide information regarding the presence of intruders or potential threat objects [6]. For military applications, such images can also provide terrain clues for helicopter navigation. Visible light images provide high-resolution structural information based on the way in which light is reflected. Thus, the fusion of thermal/infrared and visible images can be used to aid navigation, concealed weapon detection, and surveillance/border patrol by humans or automated computer vision security systems [7]. In remote sensing applications, the fusion of multispectral low-resolution remote sensing images with a high-resolution panchromatic image can yield a high-resolution multispectral image with good spectral and spatial characteristics $[8,9]$. As a visible light image is taken at a given focal point, certain objects in the image may be in focus while others may be blurred and out of focus. For digital camera applications and consumer use, the fusion of images taken at different focal points can essentially create an image having multiple focal points in which all objects in the scene are in focus [10].

The most basic image fusion approaches include spatial domain techniques using simple averaging, Principal Component Analysis (PCA) [11], and the Intensity-HueSaturation (IHS) transformation [12]. However, such methods do not incorporate aspects of the human visual system in their formulation. It is well known that the human visual system is particularly sensitive to edges at their various scales [13]. Based on this fact, multiresolution image fusion techniques have been proposed in order to yield more visually accurate fusion results. These approaches decompose image signals into lowpass and highpass coefficients via a multiresolution decomposition scheme, fuse lowpass and highpass coefficients according to specific fusion rules, and perform an inverse transform to yield the final fusion result. The use of different fusion rules for lowpass and highpass coefficients provides a means of yielding fusion results inspired by the human visual system. Pixel-based image fusion algorithms fuse detail coefficients pixels individually based on either selection or weighted averaging. Motivated by the fact that applications requiring image fusion are interested in integrating information at the feature level, region-based image fusion algorithms use segmentation to extract regions corresponding to perceived objects from the source images and fuse regions according to a region activity measure [1]. Because of their general formulations, both pixel- and region-based fusion rules can be adopted using any multiresolution decomposition technique, allowing for a convenient means of comparing the performance of multiresolution decomposition schemes for image fusion while keeping the fusion rules constant. The most common multiresolution decomposition schemes for image fusion have been the pyramid transforms and wavelet transforms. Particularly, pixel- and region-based image fusion algorithms using the Laplacian Pyramid (LP) [14], Discrete Wavelet Transform (DWT) [15], and Stationary Wavelet Transform (SWT) [16] have been proposed.

Although much of the research in image fusion has strived to formulate effective image fusion techniques which are consistent with the human visual system, the mentioned multiresolution decomposition schemes and their respective image fusion algorithms are implemented using standard arithmetic operators which are not suitable for processing images. Conversely, the Logarithmic Image Processing (LIP) model was proposed to provide a nonlinear framework for visualizing images using a mathematically rigorous arithmetical structure specifically designed for image manipulation [17]. The LIP model views images in terms of their graytone functions, which are interpreted as absorption filters. It processes graytone functions using a new arithmetic which replaces standard arithmetical operators. The resulting set of arithmetic operators can be used to process images based on a physically relevant image formation model. The model makes use of a logarithmic isomorphic transformation, consistent with the fact that the human visual system processes light logarithmically. The model has also shown to satisfy Weber's Law, which quantifies the human eye's ability to perceive intensity differences for a given background intensity [18]. As a result, image enhancement [19], edge detection [20], and image restoration [21] algorithms utilizing the LIP model have yielded better results.

However, an unfortunate consequence of the LIP model for general practical purposes is that the dynamic range of the processed image data is left unchanged causing information loss and signal clipping. Moreover, specifically for image fusion purposes, the combination of source images in regions of vastly different mean intensity yields visually poor results even though their processing is motivated by a relevant physical model. It is therefore advantageous to formulate a generalized image processing framework which is able to effectively unify the LIP and standard processing frameworks into a single framework. Consequently, the Parameterized Logarithmic Image Processing (PLIP) model was formulated. The PLIP model is a generalization of the LIP model which attempts to overcome the mentioned shortcomings of the standard processing and LIP models and can yield visually more pleasing outputs [22]. A mathematical analysis shows that in fact LIP and standard mathematical operators are instances of the generalized PLIP framework. Adaptations of edge detection [23] and image enhancement algorithms [24] using the PLIP model have demonstrated the improved performance achieved by the parameterized framework. In this paper, we investigate the use of the PLIP model for image fusion applications. New multiresolution decomposition schemes and image fusion rules using the PLIP model are introduced, and consequently, new pixeland region-based image fusion algorithms using the PLIP model are proposed.

The remainder of this paper is organized as follows. Section 2 describes the PLIP model and analyzes its properties. Section 3 introduces the new parameterized logarithmic multiresolution image decomposition schemes. Section 4 introduces the new image fusion algorithms using the PLIP model by combining the new decomposition schemes with new parameterized logarithmic image fusion rules. Section 5 describes the Piella and Heijmans $Q_{W}$ quality metric [25] used to quantitatively assess image fusion quality. Section 6 compares the proposed image fusion algorithms with existing standards via computer simulations. Section 7 draws conclusions based on the presented experimental results. 
TABLE 1: Summary of the LIP and PLIP model mathematical operators.

\begin{tabular}{lcc}
\hline & LIP model & PLIP model \\
\hline Graytone & $g=M-I$ & $g=\mu-I$ \\
Addition & $g_{1} \triangle g_{2}=g_{1}+g_{2}-\frac{g_{1} g_{2}}{M}$ & $g_{1} \tilde{\oplus} g_{2}=g_{1}+g_{2}-\frac{g_{1} g_{2}}{\gamma}$ \\
Subtraction & $g_{1} \triangle g_{2}=M \frac{g_{1}-g_{2}}{M-g_{2}}$ & $g_{1} \tilde{\Theta} g_{2}=k \frac{g_{1}-g_{2}}{k-g_{2}}$ \\
$\begin{array}{l}\text { Scalar } \\
\text { Multiplication }\end{array}$ & $c \triangle g_{1}=M-M\left(1-\frac{g_{1}}{M}\right)^{c}$ & $c \tilde{\theta} g_{1}=\tilde{\varphi}^{-1}\left(c \tilde{\varphi}\left(g_{1}\right)\right)=\gamma-\gamma\left(1-\frac{g_{1}}{\gamma}\right)^{c}$ \\
$\begin{array}{l}\text { Isomorphic } \\
\text { Transformation } \\
\text { Graytone }\end{array}$ & $\varphi(g)=-M \ln \left(1-\frac{g}{M}\right), \varphi^{-1}(g)=-M\left[1-\exp \left(-\frac{g}{M}\right)\right]$ & $\tilde{\varphi}(g)=-\lambda \cdot \ln ^{\beta}\left(1-\frac{g}{\lambda}\right), \tilde{\varphi}^{-1}(g)=\lambda\left[1-\exp \left(\frac{-g}{\lambda}\right)^{1 / \beta}\right]$ \\
$\begin{array}{l}\text { Multiplication } \\
\text { Convolution }\end{array}$ & $g_{1} \triangle g_{2}=\varphi^{-1}\left(\varphi\left(g_{1}\right) \varphi\left(g_{2}\right)\right)$ & $g_{1} \tilde{\bullet} g_{2}=\tilde{\varphi}^{-1}\left(\tilde{\varphi}\left(g_{1}\right) \tilde{\varphi}\left(g_{2}\right)\right)$ \\
\hline
\end{tabular}

\section{Parameterized Logarithmic Image Processing}

In this section, the PLIP model is reviewed. The model extends the concept of nonlinear image processing frameworks initially proposed by Jourlin and Pinoli [17] in the form of the LIP model. The advantageous properties of the added parameterization relative to the LIP model are analyzed.

The PLIP model generalizes the LIP model, which processes images as absorption filters known as graytones based on $M$, the maximum value of the range of $I$. The original LIP model is characterized by its isomorphic transformation, which mathematically emulates the relevant nonlinear physical model which the LIP model is based on. A new set of LIP mathematical operators, namely, addition, subtraction, and scalar multiplication, are consequently defined for graytones $g_{1}$ and $g_{2}$ and scalar constant $c$ in terms of this isomorphic transformation, thus replacing traditional mathematical operators with nonlinear operators which attempt to characterize the nonlinearity of image arithmetic. For example, LIP addition emulates the intensity image projected onto a screen when a uniform light source is filtered by two graytones placed in series. Subsequently, LIP convolution is also defined for a graytone $g$ and filter $w$ [26].

Table 1 summarizes and compares the LIP and PLIP mathematical operators. In its most general form, the PLIP model generalizes graytone calculation, arithmetic operations, and the isomorphic transformation independently, giving rise to the model parameters $\mu, \gamma, k, \lambda$, and $\beta$. To reduce the number of parameters needed for image fusion, this paper considers the specific instance in which $\mu=$ $M, \gamma=k=\lambda$, and $\beta=1$, effectively resulting in a single model parameter $\gamma$. In this case, The PLIP model generalizes the isomorphic transformation which defines the LIP model by accordingly choosing values for $\gamma$. Practically, for images in $[0, M)$, the value of $\gamma$ can either be chosen such that $\gamma \geq M$ for positive $\gamma$ or can take on any negative value. The resulting PLIP mathematical operators based on the parameterized isomorphic transformation can be subsequently derived.
2.1. Properties. The PLIP properties to be discussed refer to the specific instance of the PLIP model in which $\mu=M, \gamma=$ $k=\lambda$, and $\beta=1$. Similar intuitions are deduced for the more general cases.

1. The PLIP model operators revert to the LIP model operators with $\gamma=M$.

2. It can be shown that

$$
\lim _{|\gamma| \rightarrow \infty} \tilde{\varphi}(a)=\lim _{|\gamma| \rightarrow \infty} \tilde{\varphi}^{-1}(a)=a .
$$

Since $\tilde{\varphi}$ and $\tilde{\varphi}^{-1}$ are continuous functions, the PLIP model operators revert to arithmetic operators as $|\gamma|$ approaches infinity, and therefore, the PLIP model approaches standard linear processing of graytone functions as $|\gamma|$ approaches infinity. Depending on the nature of the algorithm, an algorithm which utilizes standard linear processing operators can be found to be an instance of an algorithm using the PLIP model with $\gamma=\infty$.

3. The PLIP model can generate intermediate cases between LIP operators and standard operators by choosing $\gamma$ in the range $(M, \infty)$.

4. For input graytones in $[0, M)$, the range of PLIP addition and multiplication with $\gamma$ in $[M, \infty]$ is $[0, \gamma]$.

5. For input graytones in $[0, M)$, the range of PLIP subtraction with $\gamma$ in $[M, \infty]$ is $(-\infty, \gamma]$.

6. It can be shown that the PLIP operators obey the associative, commutative, and distributive laws and unit identities.

7. The operations satisfy Jourlin and Pinoli's [17] requirements for image processing frameworks and an additional 5th one. Namely, (1) the image processing framework must be based on a physically relevant image formation model. (2) The mathematical operations must be consistent with the physical nature of images. (3) The operations must be computationally effective. (4) The framework must be practically fruitful. (5) The framework must minimize the loss of information. 
The 5th requirement essentially states that when visually "good" images are processed, the output must also be visually "good" [22]. The PLIP model satisfies the requirements by selecting values of $\gamma$ which expands the dynamic range of outputs in order to minimize information loss while also retaining nonlinear, logarithmic functionality according to a physical model. Thus, for positive $\gamma$, the PLIP model physically provides a balance between the standard linear processing model and the LIP model. Conversely, negative values of $\gamma$ may be selected for cases in which added brightness is needed to yield more visually pleasing results.

\section{Parameterized Logarithmic Multiresolution Image Decomposition Schemes}

Image fusion algorithms using the PLIP model require a mathematical formulation of multiresolution decomposition schemes and fusion rules in terms of the model. In this section, we introduce new parameterized logarithmic multiresolution decomposition schemes and fusion rules. It should be noted that they are defined for graytones. Therefore, images are converted to graytones before PLIPbased operations are performed and converted from graytone values to grayscale values after PLIP-based operations are performed.

3.1. Parameterized Logarithmic Laplacian Pyramid. The LP, originally proposed by Burt and Adelson [14], uses the Gaussian Pyramid to provide a multiresolution image representation for an image $I$. Each analysis stage consists of lowpass filtering, downsampling, interpolating, and differencing steps in order to generate the approximation coefficients $y_{0}^{(n)}$ and detail coefficients $y_{1}^{(n)}$ at scale $n$. According to the PLIP model, the approximation coefficients for the Parameterized Logarithmic Laplacian Pyramid (PL-LP) of a graytone $g$ at a scale $n>0$ are generated by

$$
\tilde{y}_{0}^{(n)}=\left[w \tilde{*} \tilde{y}_{0}^{(n-1)}\right]_{\downarrow 2}
$$

where $\tilde{y}_{0}^{(n)}=g, \tilde{*}$ denotes PLIP convolution, and $w$ is a $2 \mathrm{D}$ lowpass filter. For example, $w$ can be defined by

$$
w=\frac{1}{256}\left[\begin{array}{ccccc}
1 & 4 & 6 & 4 & 1 \\
4 & 16 & 24 & 16 & 4 \\
6 & 24 & 36 & 24 & 6 \\
4 & 16 & 24 & 16 & 4 \\
1 & 4 & 6 & 4 & 1
\end{array}\right]
$$

The detail coefficients at scale $n$ are consequently calculated as a weighted difference between successive levels of the Gaussian Pyramid and are given by

$$
\tilde{y}_{1}^{(n)}=\tilde{y}_{0}^{(n)} \tilde{\Theta}(4 w) \tilde{*}\left[\tilde{y}_{0}^{(n+1)}\right]_{\uparrow 2} .
$$

The inverse procedure begins from the approximation coefficient at the high decomposition level $N$. Each synthesis level reconstructs approximation coefficients at a scale $i<N$ by each synthesis level by

$$
\tilde{y}_{0}^{(n)}=\tilde{y}_{1}^{(n)} \oplus(4 w) \tilde{*}\left[\tilde{y}_{0}^{(n+1)}\right]_{\uparrow 2} .
$$

\subsection{Parameterized Logarithmic Discrete Wavelet Transform.} The 2D separable DWT uses a quadrature mirror set of 1D analysis filters, $g$ and $h$, and synthesis filters, $\hat{g}$ and $\hat{h}$, to provide a multiresolution scheme for an image $I$ with added directionality relative to the LP [15]. The DWT is able to provide perfect reconstruction while using critical sampling. Each analysis stage consists of filtering along rows, downsampling along columns, filtering along columns, and downsampling along rows in order to generate the approximation coefficient subband $y_{0}^{(n)}$ and detail coefficient subbands $y_{1}^{(n)}, y_{2}^{(n)}$, and $y_{3}^{(n)}$ oriented horizontally, vertically, and diagonally, respectively, at scale $n$. The synthesis procedure begins from the wavelet coefficients at the highest decomposition level $N$. Filtering and upsampling steps are performed in order to perfectly reconstruct the image signal. According to the PLIP model, the Parameterized Logarithmic Discrete Wavelet Transform (PL-DWT) at graytone $g$ at a decomposition level $n>0$ is calculated by making use of the parameterized isomorphic transformation and is defined by

$$
\widetilde{W}_{\mathrm{DWT}}\left(\tilde{y}_{0}^{(n)}\right)=\tilde{\varphi}^{-1}\left(W_{\mathrm{DWT}}\left(\tilde{\varphi}\left(\tilde{y}_{0}^{(n)}\right)\right)\right)
$$

where $\tilde{y}_{0}^{(0)}=g$. Similarly, each synthesis level reconstructs approximation coefficients at a scale $i<N$ by

$$
\widetilde{W}_{\text {DWT }}^{-1}\left(\widetilde{W}_{\text {DWT }}\left(\tilde{y}_{0}^{(n)}\right)\right)=\widetilde{\varphi}^{-1}\left(W_{\text {DWT }}^{-1}\left(\widetilde{\varphi}\left(\widetilde{W}_{\text {DWT }}\left(\tilde{y}_{0}^{(n)}\right)\right)\right)\right)
$$

3.3. Parameterized Logarithmic Stationary Wavelet Transform. Both the DWT and LP are shift-variant due to the downsampling step which they employ. Therefore, the alteration of transform coefficients may introduce artifacts when processed using the DWT and to a lesser extent, the LP. It can introduce artifacts into the fusion results particularly for cases in which source images are misregistered. The SWT is a shift-invariant, redundant wavelet transform which attempts to reduce artifact effects by upsampling analysis filters rather than downsampling approximation images at each level of decomposition [27]. According to the PLIP model, the forward and inverse Parameterized Logarithmic Stationary Wavelet Transform (PL-SWT) for a graytone $g$ at a decomposition level $n>0$ is calculated by

$$
\begin{gathered}
\widetilde{W}_{\text {SWT }}\left(\widetilde{y}_{0}^{(n)}\right)=\widetilde{\varphi}^{-1}\left(W_{\text {SWT }}\left(\widetilde{\varphi}\left(\widetilde{y}_{0}^{(n)}\right)\right)\right) \\
\widetilde{W}_{\text {SWT }}^{-1}\left(\widetilde{W}_{\text {SWT }}\left(\widetilde{y}_{0}^{(n)}\right)\right)=\widetilde{\varphi}^{-1}\left(W_{\text {SWT }}^{-1}\left(\widetilde{\varphi}\left(\widetilde{W}_{\text {SWT }}\left(\widetilde{y}_{0}^{(n)}\right)\right)\right)\right) .
\end{gathered}
$$




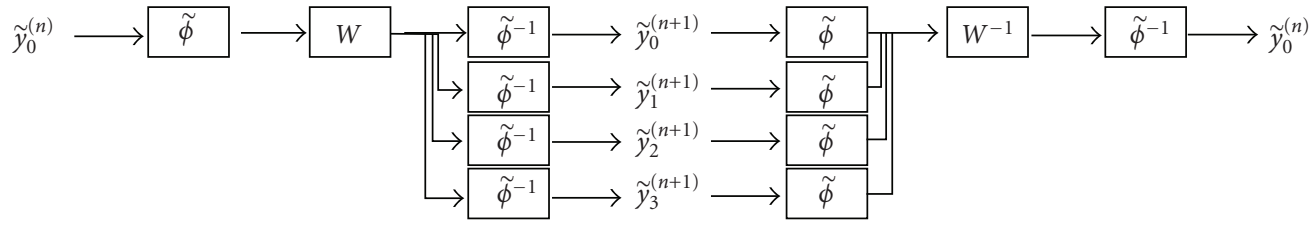

FIgURe 1: Parameterized Logarithmic Wavelet Transform analysis and synthesis.

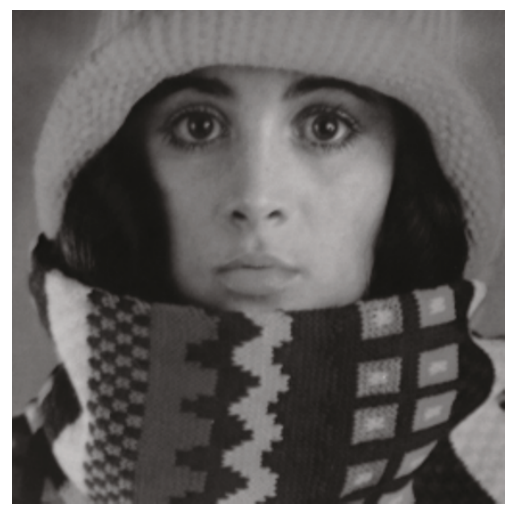

(a)

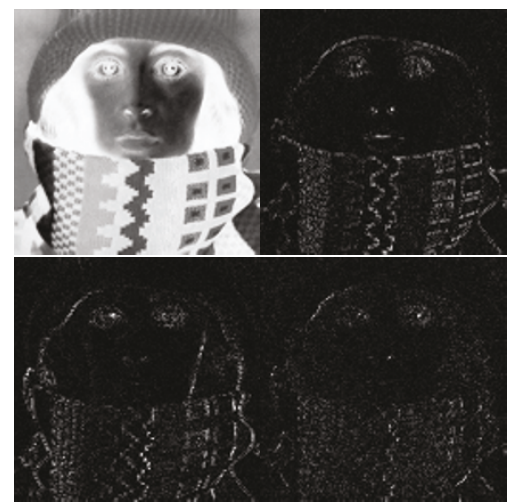

(d)

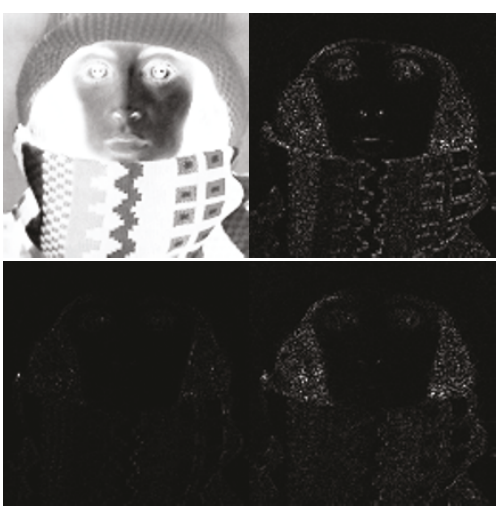

(b)
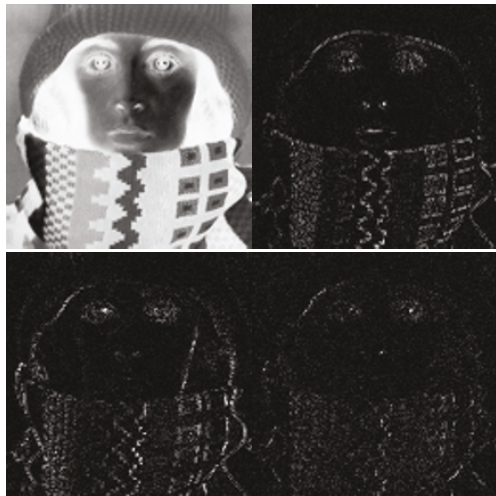

(e)

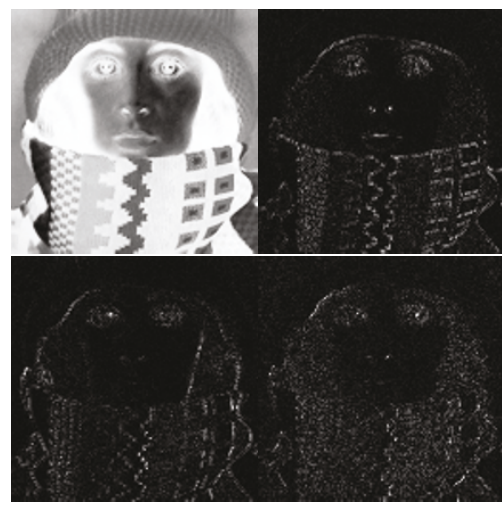

(c)

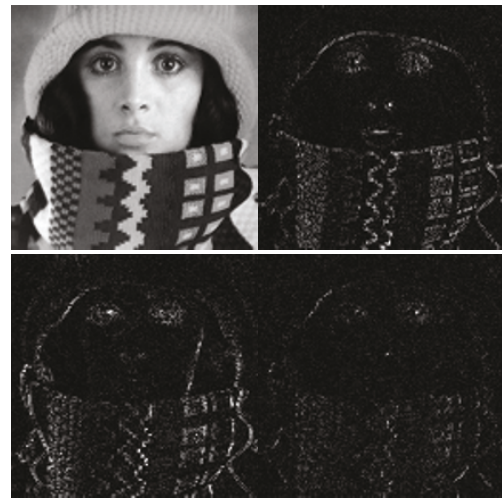

(f)

FIGURE 2: (a) Original “Trui” image, top-left: approximation subband, magnitude of top-right: horizontal subband, bottom-left: vertical subband, bottom-right: diagonal subband magnitude of horizontal subband using the SWT and PLIP model operators with (b) $\gamma=256$ (LIP model case), (c) $\gamma=300$, (d) $\gamma=500$, (e) $\gamma=700$, and (f) standard mathematical operators.

Figure 1 illustrates the analysis and synthesis stages using PLIP wavelet transforms, where $W$ is a type of wavelet transform (e.g., DWT, SWT, etc.) with a given set of wavelet filters [28]. As the parameterized logarithmic decomposition approaches essentially make use of standard decomposition schemes with added preprocessing and postprocessing in the form of the isomorphic transformation calculations, they can be computed with minimal added computation cost.

Figure 2 illustrates the advantages yielded using parameterized logarithmic multiresolution schemes. The wavelet decomposition using $\gamma=256$ (LIP model case) predominantly extracts the hair features from the image. As $\gamma$ increases, it is particularly apparent that the hair textures are less emphasized and that the scarf, hat, and facial edges and textures are more emphasized. The wavelet decomposition using standard operators extracts the most texture and edge information from the scarf, hat, and face in the image, and close to none of the texture of the hair. Visually, it is seen that the wavelet decomposition using the PLIP model operators with $\gamma=300$ provides the best balance between extracting the hair, scarf, hat, and facial features in the image. Ultimately, the salient features which need to be extracted at each scale for further processing are task and image dependent, and thus, the PLIP model parameter can be tuned accordingly.

\section{Image Fusion Using the PLIP Model}

In addition to the new parameterized logarithmic multiresolution image decomposition schemes, we introduce new parameterized and logarithmic approximation coefficient 


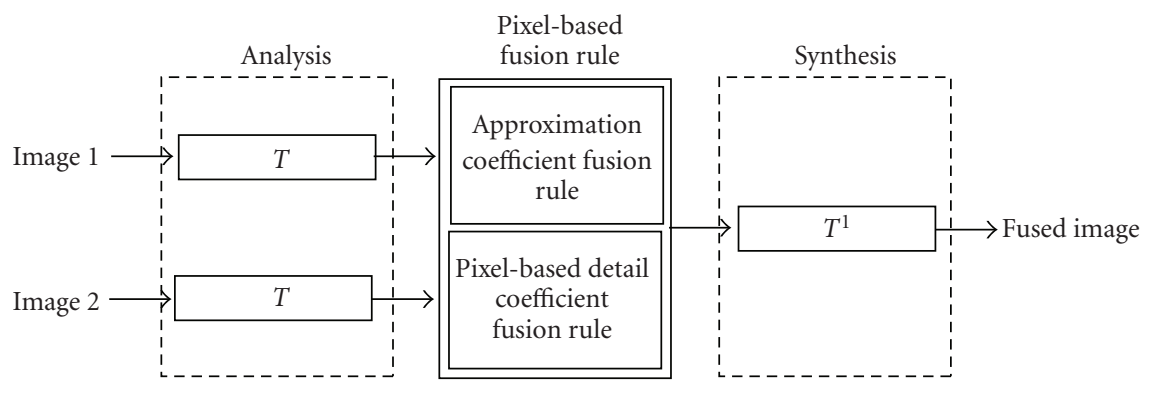

FIgURE 3: A generalized pixel-based multiresolution image fusion algorithm.

and detail coefficient fusion rules according to the PLIP model. The combination of the parameterized logarithmic image decomposition techniques and fusion rules yields a new set of image fusion algorithms which are based on the PLIP model. Consequently, due to the generalization of the PLIP operators, image fusion algorithms using LIP operators and standard operators are also encapsulated by the proposed approaches.

4.1. Parameterized Logarithmic Pixel-Based Image Fusion. A generalized pixel-based multiresolution image fusion algorithm is illustrated in Figure 3. The input source images are transformed using a given multiresolution image decomposition technique $T$. One fusion rule is used to fuse the approximation coefficients at the highest decomposition level. A second fusion rule is used to fuse the detail coefficients at each decomposition level. The resulting inverse transform yields the final fused result. Although image fusion algorithms are expected to withstand minor registration differences, the source images to be fused are assumed to be registered. Misregistered source images should be subjected to registration preprocessing steps independent to the image fusion algorithm. The approximation coefficients at the highest level of decomposition $N$ are most commonly fused via uniform averaging. This is because at the highest level of decomposition, the approximation coefficients are interpreted as the mean intensity value of the source images with all salient features encapsulated by the detail coefficient subbands at their various scales [1]. Therefore, fusing approximation coefficients at their highest level of decomposition by averaging maintains the appropriate mean intensity needed for the fusion result with minimal loss of salient features. Given $\tilde{y}_{I_{1}, 0}^{(N)}$ and $\tilde{y}_{I_{2}, 0}^{(N)}$, the approximation coefficient subbands of images $I_{1}$ and $I_{2}$, respectively, at the highest decomposition level $N$ yielded using a given parameterized logarithmic multiresolution decomposition technique, the approximation coefficients for the fused image $F$ at the highest level of decomposition using simple averaging according to the PLIP model by

$$
\tilde{y}_{F, 0}^{(N)}=\frac{1}{2} \widetilde{\otimes}\left(\tilde{y}_{I_{1}, 0}^{(N)} \widetilde{\oplus} \tilde{y}_{I_{2}, 0}^{(N)}\right)
$$

In general, an approximation coefficient fusion rule can be adapted according to the PLIP model by

$$
\tilde{y}_{F, 0}^{(N)}=\tilde{\varphi}^{-1}\left(R_{A}\left(\tilde{\varphi}\left(\tilde{y}_{I_{1}, 0}^{(N)}\right), \tilde{\varphi}\left(\tilde{y}_{I_{2}, 0}^{(N)}\right)\right)\right)
$$

where $R_{A}$ is an approximation coefficient fusion rule implemented using standard arithmetic operators. An analysis of the PLIP addition operation in Table 1 and (9) yields a simple interpretation of the effect of $\gamma$ on fusion results. Practically, $\gamma$ can be interpreted as a brightness parameter, where negative values of $\gamma$ yield brighter fusion results and positive values of $\gamma$ yield darker fusion results. This is achieved while also maintaining the fusion identity that the fusion of identical source images is the source image itself. Therefore, improved visual quality is achieved within an image fusion context and not as a result of an independent image enhancement process. The influence of the parameterization on fusion results is not limited to this naïve observation, however, as the model parameter $\gamma$ also influences the multiscale decomposition scheme and the detail coefficient fusion rule.

Conversely, the detail coefficients of the source images correspond to salient features such as lines and edges detected at various scales. Therefore, fusion rules for detail coefficients at each decomposition level should be formulated in order to preserve these features. Such fusion rules are inspired by the human visual system, which is particularly sensitive to edges. Many pixel-based detail coefficient fusion rules have been proposed. In this paper, the absolute maximum (AM) and Burt and Kolczynski (BK) pixel-based detail coefficient fusion rules are considered and formulated according to the PLIP model. The parameterized logarithmic detail coefficient fusion rules are defined according to the PLIP model by

$$
\tilde{y}_{F, i}^{(n)}=\tilde{\varphi}^{-1}\left(R_{D}\left(\tilde{\varphi}\left(\tilde{y}_{I_{1}, i}^{(n)}\right), \tilde{\varphi}\left(\tilde{y}_{I_{2}, i}^{(n)}\right)\right)\right)
$$

where $R_{D}$ is a coefficient fusion rule implemented using standard arithmetic operators.

4.1.1. Parameterized Logarithmic Absolute Maximum Detail Coefficient Fusion Rule. The AM detail coefficient fusion rule selects the detail coefficient in each subband of greatest magnitude [1]. For each of the $i$ highpass subbands at 
each level of decomposition $n$, the multiplicative weights for fusion are given by

$$
\lambda_{i}^{(n)}(k, l)= \begin{cases}1, & \left|y_{I_{1}, i}^{(n)}(k, l)\right|>\left|y_{I_{2}, i}^{(n)}(k, l)\right|, \\ 0, & \left|y_{I_{1}, i}^{(n)}(k, l)\right| \leq\left|y_{I_{2}, i}^{(n)}(k, l)\right| .\end{cases}
$$

For each of the $i$ highpass subbands at each level of decomposition $n$, the detail coefficients of the fused image $F$ are determined by

$$
y_{F, i}^{(n)}(k, l)=\lambda_{i}^{(n)}(k, l) y_{I_{1}, i}^{(n)}(k, l)+\left(1-\lambda_{i}^{(n)}(k, l)\right) y_{I_{2}, i}^{(n)}(k, l) .
$$

Accordingly, the parameterized logarithmic AM rule is yielded by (11).

4.2. Parameterized Logarithmic Burt and Kolczynski Detail Coefficient Fusion Rule. The BK detail coefficient fusion rule combines detail coefficients based on an activity measure and a match measure [29]. The activity measure for each $w \times w$ local window of each subband $i$ is calculated for each source image, given as

$$
a_{I, i}^{(n)}(k, l)=\sum_{(\Delta k, \Delta l) \in W}\left(y_{I, i}^{(n)}(k+\Delta k, l+\Delta l)\right)^{2} .
$$

The local match measure of each subband measures the correlation of each subband between source images and is given as

$$
\begin{aligned}
& m_{I_{1}, I_{2}, i}^{(n)}(k, l) \\
& =\frac{2 \sum_{(\Delta k, \Delta l) \in W}\left(y_{I_{1}, i}^{(n)}(k+\Delta k, l+\Delta l)\right)\left(y_{I_{2}, i}^{(n)}(k+\Delta k, l+\Delta l)\right)}{a_{I_{1}, i}^{(n)}(k, l)+a_{I_{2}, i}^{(n)}(k, l)} .
\end{aligned}
$$

Comparing the match measure to a threshold th determines if detail coefficients are to be combined by simple selection or by weighted averaging. The associated weights for fusion are given by

$$
\lambda_{i}^{(n)}(k, l)= \begin{cases}1, & m_{I_{1}, I_{2}, i}^{(n)}(k, l) \leq t h, \\ & a_{I_{1}, i}^{(n)}(k, l)>a_{I_{2}, i}^{(n)}(k, l), \\ 0, & m_{I_{1}, I_{2}, i}^{(n)}(k, l) \leq t h, \\ & a_{I_{1}, i}^{(n)}(k, l) \leq a_{I_{2}, i}^{(n)}(k, l), \\ \frac{1}{2}+\frac{1}{2}\left(\frac{1-m_{I_{1}, I_{2}, i}^{(n)}(k, l)}{1-T}\right), & m_{I_{1}, I_{2}, i}^{(n)}(k, l)>t h, \\ \frac{1}{2}-\frac{1}{2}\left(\frac{1-m_{I_{1}, I_{2}, i}^{(n)}(k, l)}{1-T}\right), & a_{I_{1}, i}^{(n)}(k, l)>a_{I_{2}, i}^{(n)}(k, l), \\ & m_{I_{1}, I_{2}, i}^{(n)}(k, l)>t h, \\ & a_{I_{1}, i}^{(n)}(k, l) \leq a_{I_{2}, i}^{(n)}(k, l) .\end{cases}
$$

For each of the $i$ highpass subbands at each level of decomposition $n$, the detail coefficients for the fused image $F$ are again determined by (13). Accordingly, the parameterized logarithmic BK rule is yielded by (11).

Figure 4 illustrates the fundamental themes which have been discussed so far, particularly highlighting the necessity for the added model parameterization. The $Q_{W}$ quality metric [25] included in Figure 4, whose details are to be discussed further in Section 5, implies a better fusion for a higher value of $Q_{W}$. Figure 4(c) shows that firstly, the PLIP model reverts to the LIP model with $\gamma=M=$ 256 , and secondly, that the combination of source images using this extreme case may still be visually unsatisfactory given the nature of the input images, even though the processing framework is based on a physically inspired model. Figures $4(\mathrm{~d}), 4(\mathrm{e})$, and $4(\mathrm{f})$ illustrate the way in which fusion results are affected by the parameterization, with the most improved fusion performance yielded by the proposed approach using parameterized multiresolution decomposition schemes and fusion rules relative to both the standard processing extreme and the LIP model extreme with $\gamma=430$. Namely, this result using the proposed approach has better visual contrast between roads and terrain and provides the proper base luminance to effectively differentiate between the grass and bushes. Figure 5 plots the $Q_{W}$ quality metric [25] as a function of $\gamma$ and reflects the qualitative observation indicating Figure $4(\mathrm{e})$ as the best fusion output. Lastly, Figures $4(\mathrm{~g})$ and $4(\mathrm{~h})$ show using the AM fusion rule that the PLIP operators revert to standard mathematical operators as $\gamma$ approaches infinity.

4.3. Parameterized Logarithmic Region-Based Image Fusion. Pixel-based image fusion approaches determine the detail coefficients of a fused image on a per pixel basis. Namely, they use the transform data at local neighborhoods to individually determine each detail coefficient of the ultimate fusion result. Applications which utilize image fusion schemes are by and large more interested in fusing the various objects found in the original source images. This suggests that information regarding features instead of the pixels themselves should be incorporated into the fusion process. This provides the motivation for region-based image fusion algorithms [1]. Region-based fusion algorithms use image segmentation to guide the fusion process. A generalized region-based multiresolution fusion algorithm is illustrated in Figure 6. The source images are once again first transformed using a given multiresolution decomposition scheme. They are segmented using a segmentation algorithm, yielding a shared region representation which is thereby used to aid the fusion of detail coefficients at each scale. The detail coefficients in each region at each scale are fused based on their level of activity in the given region. The fusion of approximation coefficients at the highest level of decomposition remains unchanged. The result is a more robust fusion approach which can overcome blurring effects and improve sensitivity to noise and misregistration known to pixel-based approaches. Region-based image fusion has also allowed for a broader class of fusion rules to be formulated [30]. 


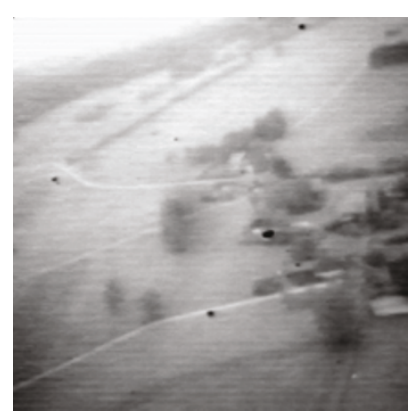

(a)

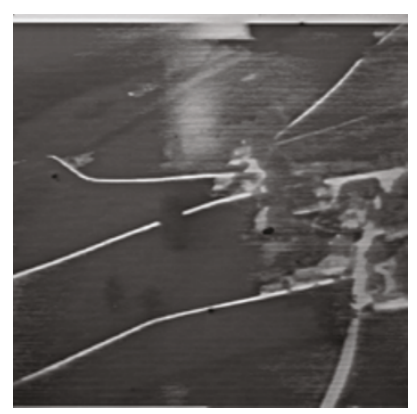

(e)

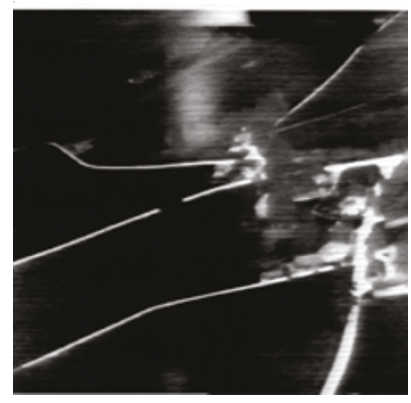

(b)

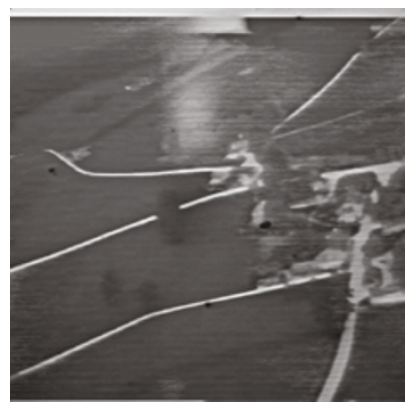

(f)

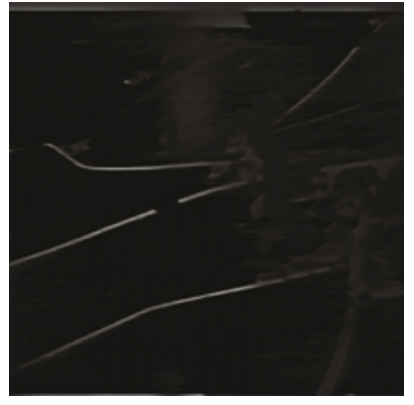

(c)

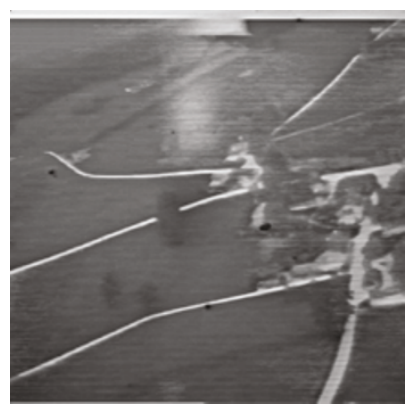

(g)

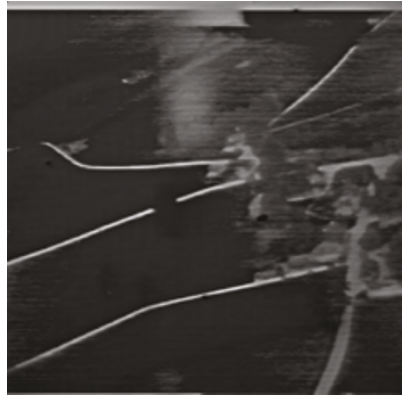

(d)

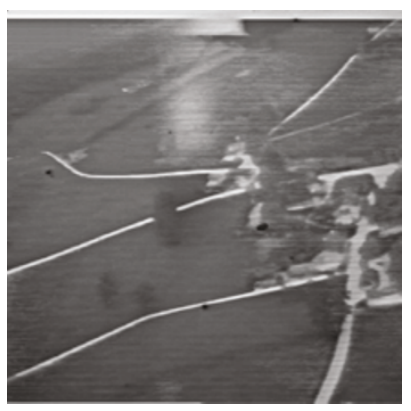

(h)

FIGURE 4: (a) and (b) Original "navigation" source images, image fusion results using the LP/AM fusion rule, and PLIP model operators with (c) $\gamma=256$ (LIP model case), $Q_{W}=0.3467$, (d) $\gamma=300, Q_{W}=0.7802$, (e) $\gamma=430, Q_{W}=0.8200$, (f) $\gamma=700, Q_{W}=0.8128$ (g) $\gamma=10^{8}$, $Q_{W}=0.7947$, and (h) standard mathematical operators, $Q_{W}=0.7947$.

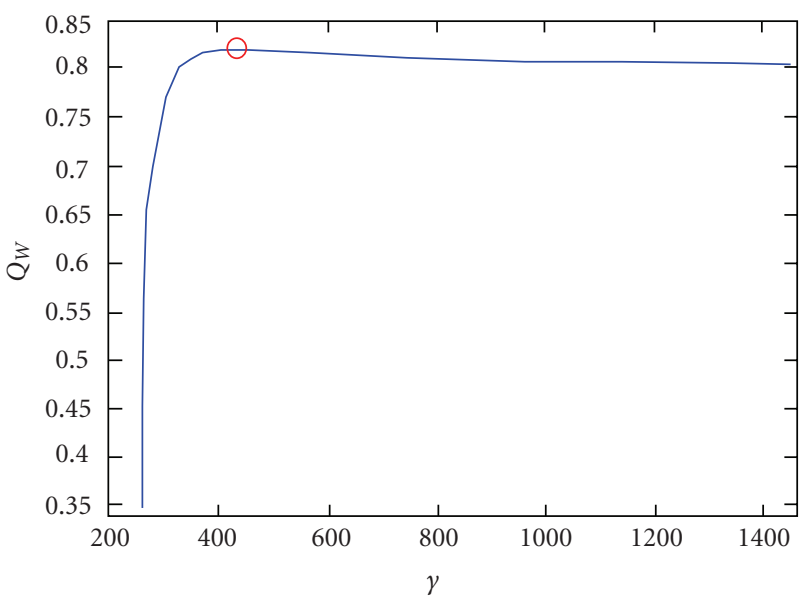

Figure 5: Plot of $Q_{W}$ versus $\gamma$ for image fusion results in Figure 4, indicating a maximum at $\gamma=430, Q_{W}=0.8200$.

The choice of segmentation algorithm used in regionbased image fusion directly affects the fusion result. Segmentation algorithms which have been used in regionbased image fusion algorithms include watershed [30], $K$-means [31], texture-based [32], pyramidal linking [1], and mean-shift segmentation [33]. In this paper, meanshift segmentation is used for all region-based approaches because of its robustness $[34,35]$. It may be substituted with another segmentation algorithm. As this paper is primarily concerned with the use of the nonlinear frameworks and multiresolution schemes for image fusion, a discussion of appropriate segmentation algorithms for image fusion is considered outside of the scope of this work. The main objective here is to extend the use of parameterized logarithmic image fusion to region-based approaches. A shared region representation for region-based image fusion purposes is yielded using mean-shift segmentation by individually segmenting each of the source images, and by then splitting overlapping regions into new regions [32]. An example of a shared region representation yielded using mean-shift segmentation is shown in Figure 7. To maintain consistency in segmentation results across different scales, successive downsampling is performed to yield a shared region representation at each level of decomposition based on the image decomposition scheme used for image fusion [33].

4.3.1. Region-Based Detail Coefficient Fusion Rules. Most any fusion rule formulated for pixel-based fusion can be easily formulated in terms of regions. The extension to regions merely involves calculating activity measures, match measures, and fusion weights for each region $R$ instead of each pixel [1]. For experimental purposes, the activity measure for each region of each subband $i$ of each source image is calculated by

$$
a_{I, i}^{(n)}(R)=\sum_{(k, l) \in R}\left(y_{I, i}^{(n)}(k, l)\right)^{2},
$$




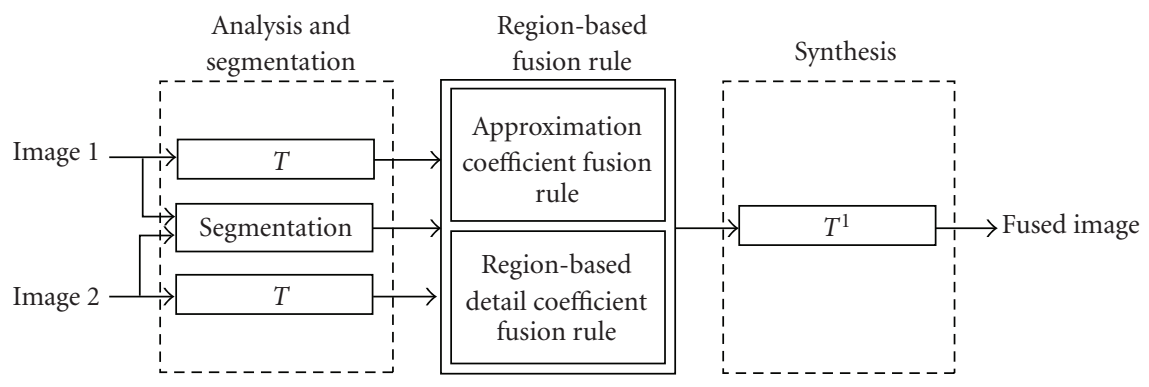

Figure 6: A generalized region-based multiresolution image fusion algorithm.

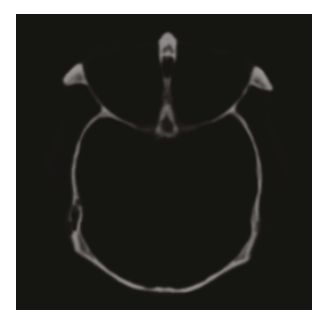

(a)

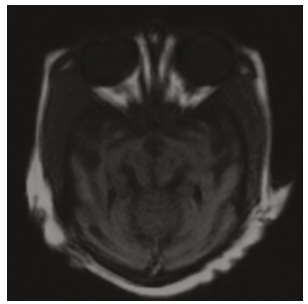

(b)

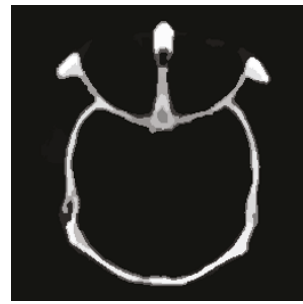

(c)

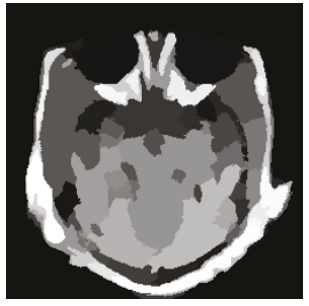

(d)

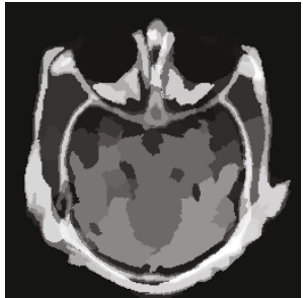

(e)

FIgURE 7: (a) and (b) Original "brain" source images, (c) mean-shift segmentation result of (a), (d) mean-shift segmentation result of (b), (e) shared region representation for region-based image fusion.

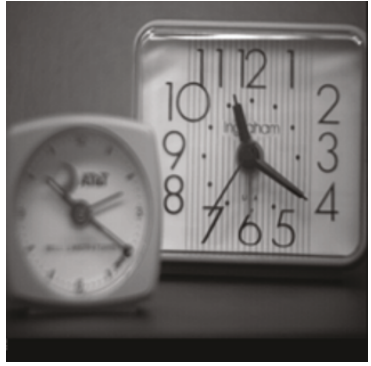

(a)

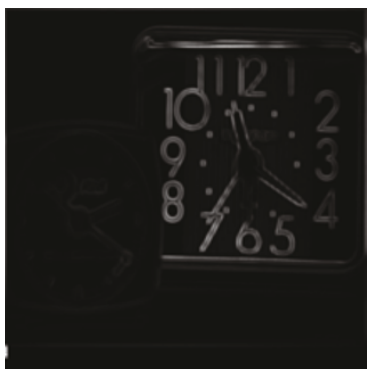

(c)

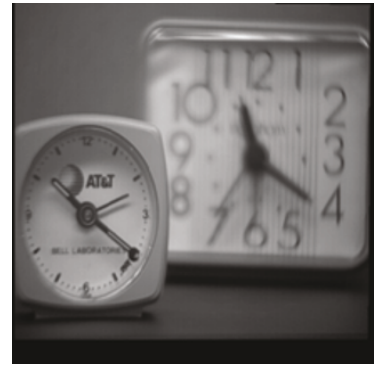

(b)

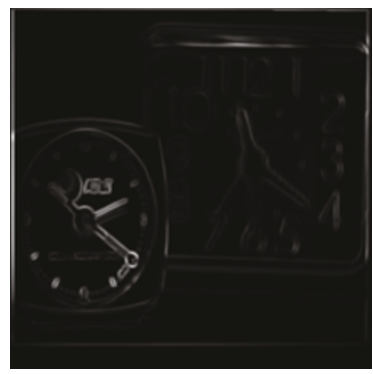

(d)
FIGURE 8: (a) and (b) Original "clock" source images, respective weights $(\mathrm{c}) c \cdot \lambda$ and $(\mathrm{d}) c \cdot(1-\lambda)$ used for image fusion quality assessment.

where $|R|$ is the area of the region $R$. Similarly, the match measure $m_{I_{1}, I_{2}, i}^{(n)}(R)$ and the multiplicative fusion weight $\lambda_{i}^{(n)}(R)$ for each region of each subband $i$ can be defined based on the fusion rule of choice. For experimental purposes, fusion weights are defined according to a regionbased absolute maximum selection rule, hereby referred to as RB, by

$$
\lambda_{i}^{(n)}(R)= \begin{cases}1, & \left|a_{I_{1}, i}^{(n)}(R)\right|>\left|a_{I_{2}, i}^{(n)}(R)\right|, \\ 0, & \left|a_{I_{1}, i}^{(n)}(R)\right| \leq\left|a_{I_{2}, i}^{(n)}(R)\right| .\end{cases}
$$

For each of the $i$ highpass subbands at each level of decomposition $n$, the detail coefficients of the fused image $F$ in each region $R$ are determined by

$$
y_{F, i}^{(n)}(R)=\lambda_{i}^{(n)}(R) y_{I_{1}, i}^{(n)}(R)+\left(1-\lambda_{i}^{(n)}(R)\right) y_{I_{2}, i}^{(n)}(R) .
$$

The parameterized logarithmic region-based image fusion rule is defined according to the PLIP model by (11).

\section{Quantitative Image Fusion Quality Assessment}

Objective performance assessment of image fusion quality is still an open problem requiring more research in order to provide valuable objective evaluation [1]. The metrics proposed by Xydeas and Petrović [36] and Piella and Heijmans [25] tend to favor fusion results which transfer more edge information into fusion results and are therefore vulnerable to noisy test cases. Conversely, mutual-information-based metrics [37] tend to favor fusion approaches which transfer relatively less edge information but are less sensitive to noise, 


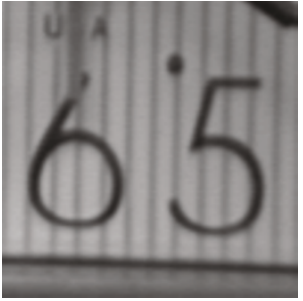

(a)

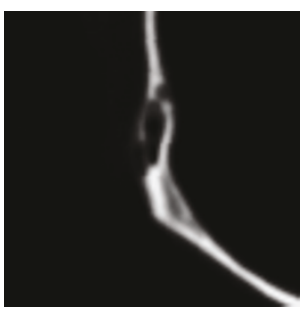

(f)

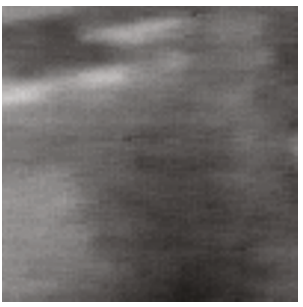

$(\mathrm{k})$

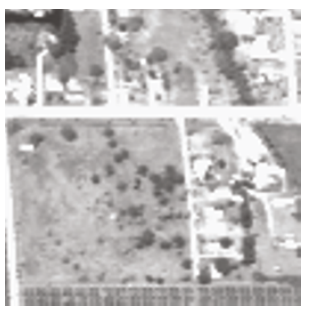

(p)

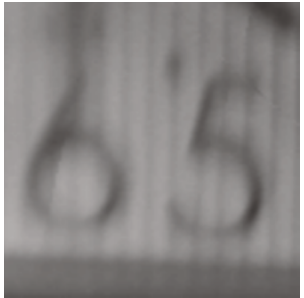

(b)

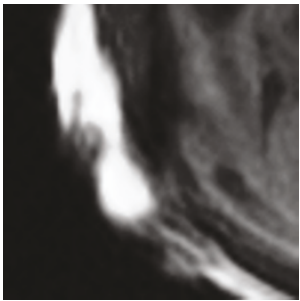

(g)

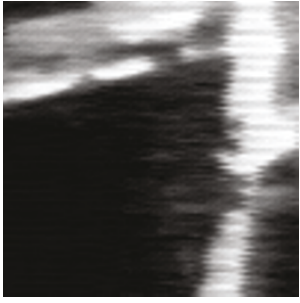

(1)

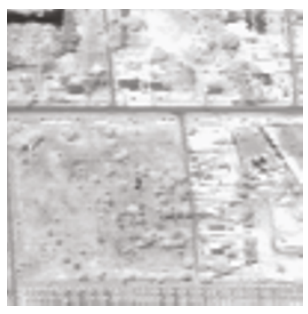

(q)

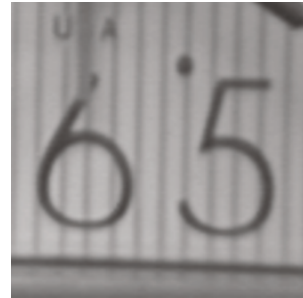

(c)

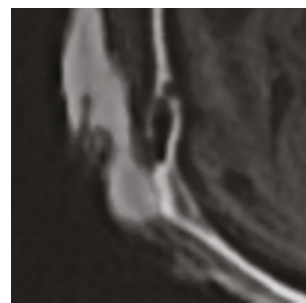

(h)

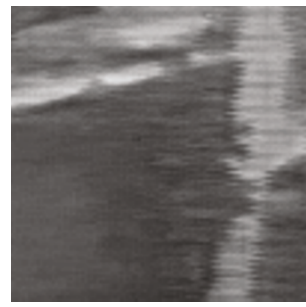

(m)

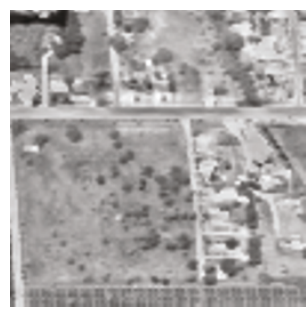

(r)

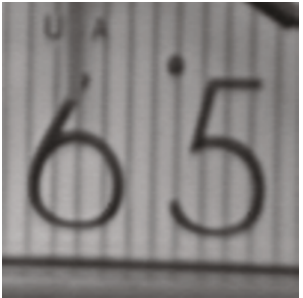

(d)

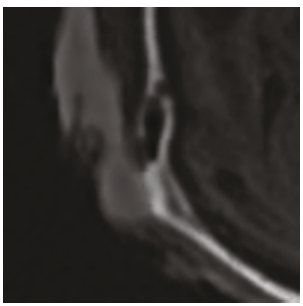

(i)

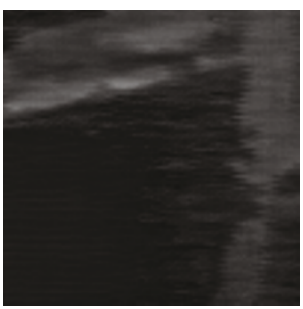

(n)

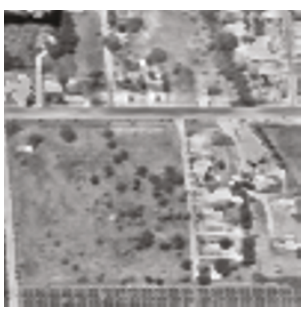

(s)

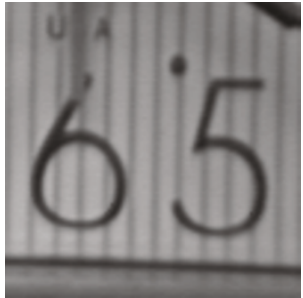

(e)

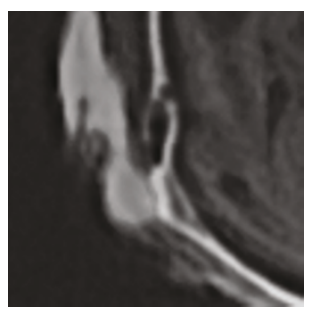

(j)

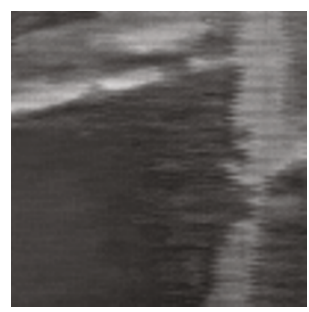

(o)

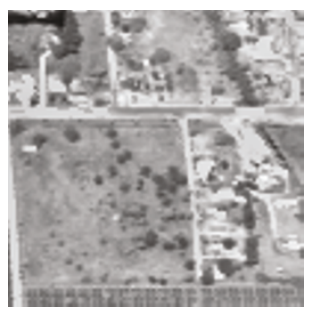

(t)

Figure 9: Zoomed regions of (a)and (b) Original "clock" source images, image fusion results using (c) LP and RB, (d) LIP-LP and RB, (e) PL-LP and RB, (f) and (g) original "brain" source images, image fusion results using (h) SWT and RB, (i) LIP-SWT and RB, (j) PL-SWT and $\mathrm{RB}(\mathrm{k})$ and (l) original "navigation" source images, image fusion results using (m) DWT and AM, (n) LIP-DWT and AM, (o) PL-DWT and $\mathrm{AM}(\mathrm{p})$ and (q) original "remote sensing" source images, image fusion results using (r) SWT and BK, (s) LIP-SWT and BK, (t) PL-SWT and BK.

such as region-based and even simple averaging approaches [25]. Nonetheless, to gain objective perspective not on the fusion rule or standard decomposition scheme of choice, but rather the improvement of fusion results using the PLIP model, fusion results are assessed quantitatively using the Piella and Heijmans image fusion quality metric. The metric measures fusion quality based on how much the fusion result reflects the original source images. Bovik's quality index [38] is used to relate the fused result to its original source images. The quality index $Q_{0}$ proposed by Bovik to measure the similarity between two sequences $x$ and $y$ is given by

$$
Q_{0}=\frac{\sigma_{x y}}{\sigma_{x} \sigma_{y}} \cdot \frac{2 \mu_{x} \mu_{y}}{\mu_{x}^{2}+\mu_{y}^{2}} \cdot \frac{2 \sigma_{x} \sigma_{y}}{\sigma_{x}^{2}+\sigma_{y}^{2}},
$$

where $\sigma_{x}$ and $\sigma_{y}$ are the sample standard deviations of $x$ and $y$, respectively, $\sigma_{x y}$ is the sample covariance of $x$ and $y$, and $\mu_{x}$ and $\mu_{y}$ are the sample means of $x$ and $y$, respectively. For two images $I$ and $F$, a sliding window technique is utilized to calculate the quality index $Q_{0}(I, F \mid w)$ at each local 


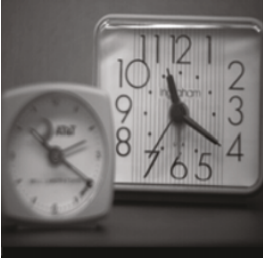

(a)
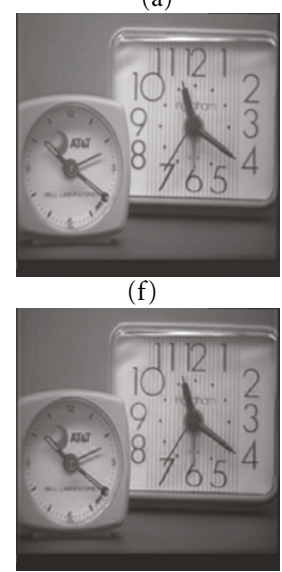

(1)

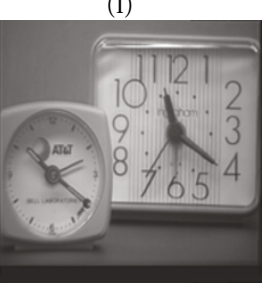

(r)

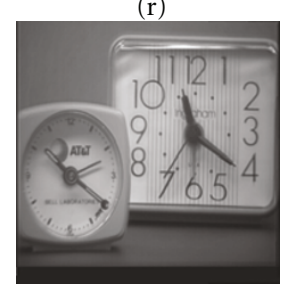

$(\mathrm{x})$

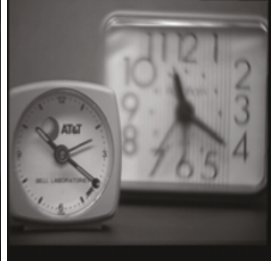

(b)

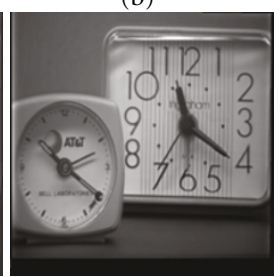

(g)

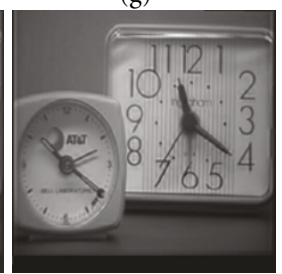

(m)

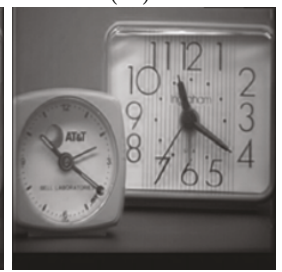

(s)

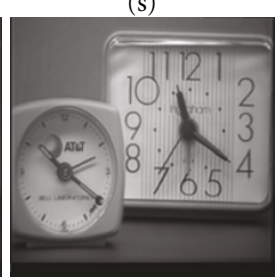

(y)

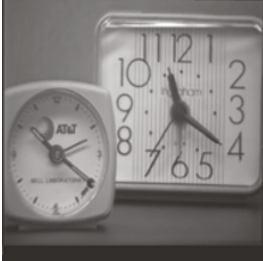

(c)

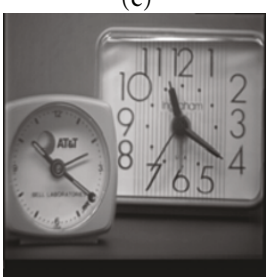

(h)

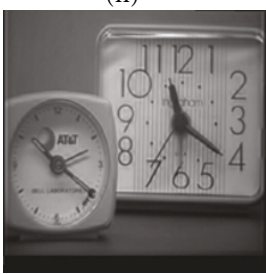

(n)

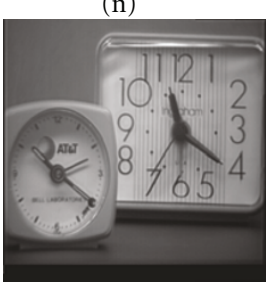

(t)

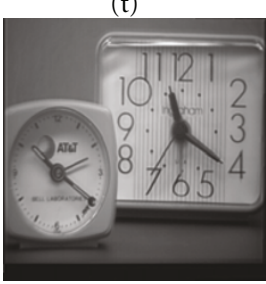

(z)

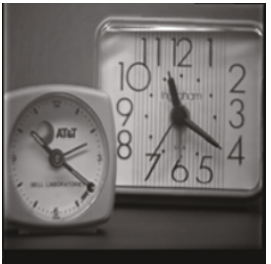

(d)

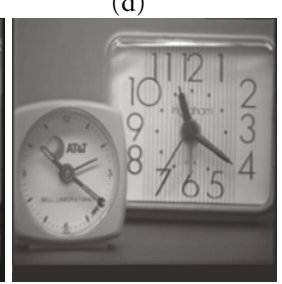

(i)

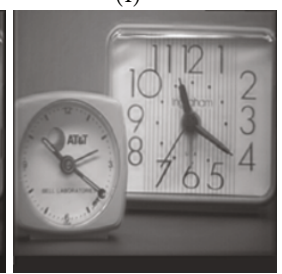

(o)

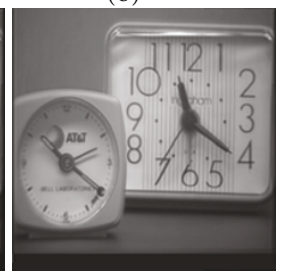

(u)

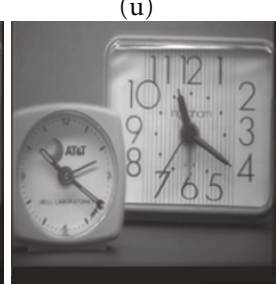

(aa)

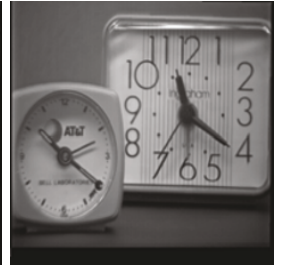

(e)

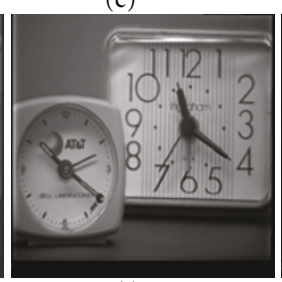

(j)

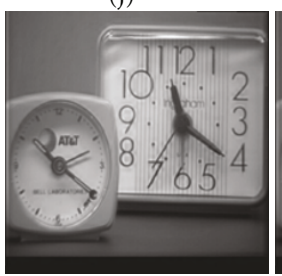

(p)

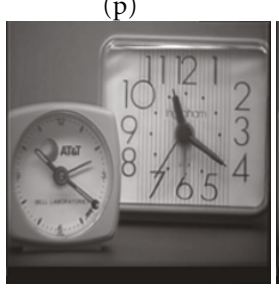

(v)

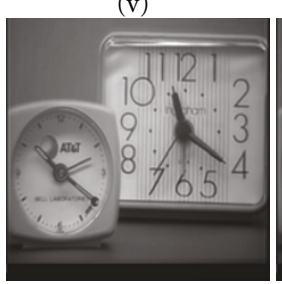

(bb)

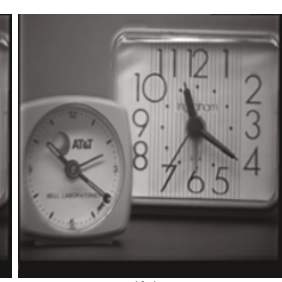

(k)

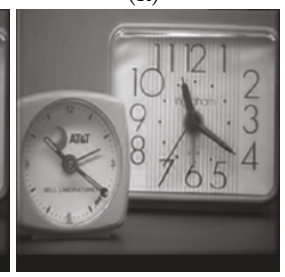

(q)

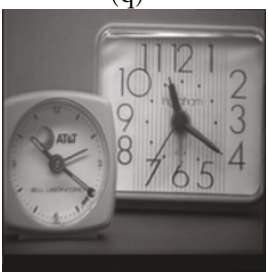

(w)

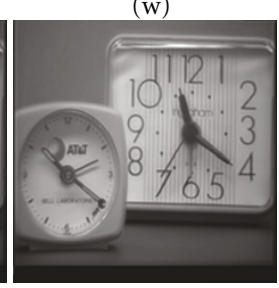

(cc)

Figure 10: (a) and (b) Original "clock" source images, image fusion results using (c) LP/AM, (d) LIP-LP/AM, (e) PL-LP/AM, (f) LP/BK, (g) LIP-LP/BK, (h) PL-LP/BK, (i) LP/RB, (j) LIP-LP/RB, (k) PL-LP/RB, (l) DWT/AM, (m) LIP-DWT/AM, (n) PL-DWT/AM, (o) DWT/BK, (p) LIP-DWT/BK, (q) PL-DWT/BK, (r) DWT/RB, (s) LIP-DWT/RB, (t) PL-DWT/RB, (u) SWT/AM, (v) LIP-SWT/AM, (w) PL-SWT/AM, (x) SWT/BK, (y) LIP-SWT/BK, (z) PL-SWT /BK, (aa) SWT/RB, (bb) LIP-SWT/RB, (cc) PL-SWT/RB.

$w \times w$ window. The average of these quality indexes is used to measure the similarity between $I$ and $F$, and is given by

$$
Q_{0}(I, F)=\frac{1}{|W|} \sum_{w \in W} Q_{0}(I, F \mid w) .
$$

The resulting similarity index ranges from 0 to 1 , with two identical images yielding a $Q_{0}$ equal to 1 . Defining $s(I \mid w)$ as the saliency, and in this case, the variance of the image $I$ in a local window $w \times w$ window, the quality of the fused result can be assessed by first calculating local weights $\lambda(w)$ for the source images $I_{1}$ and $I_{2}$, given by

$$
\lambda(w)=\frac{s\left(I_{1} \mid w\right)}{s\left(I_{1} \mid w\right)+s\left(I_{2} \mid w\right)}
$$

and then calculating the fusion quality index $Q\left(I_{1}, I_{2}, F\right)$ for the fused result $F$ by

$$
\begin{aligned}
& Q\left(I_{1}, I_{2}, F\right) \\
& =\frac{1}{|W|} \sum_{w \in W}\left(\lambda(w) Q_{0}\left(I_{1}, F \mid w\right)+(1-\lambda(w)) Q_{0}\left(I_{2}, F \mid w\right)\right) .
\end{aligned}
$$

The metric assesses fusion quality by calculating the local quality indexes between the fused image and the two source images, and weighting them according to the local saliency between the source images. To better reflect the human visual system, another weight is added to give more weight to 


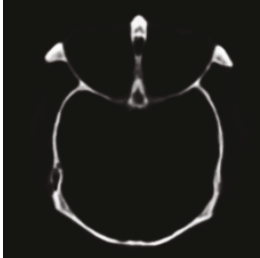

(a)

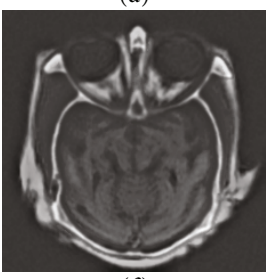

(f)

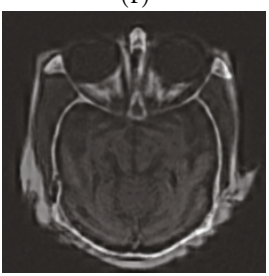

(1)

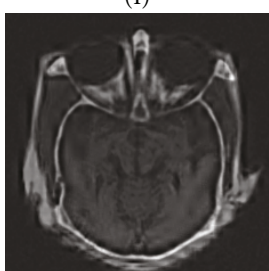

(r)

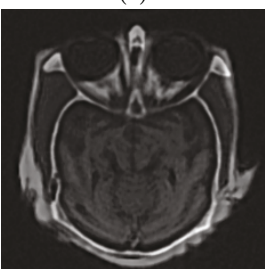

(x)

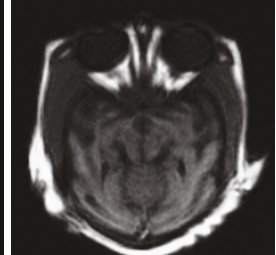

(b)

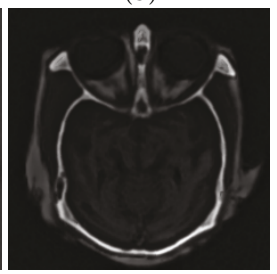

(g)

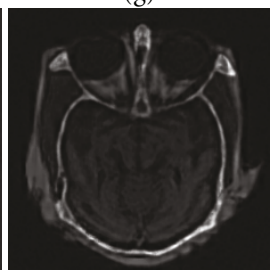

(m)

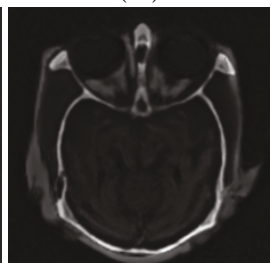

(s)

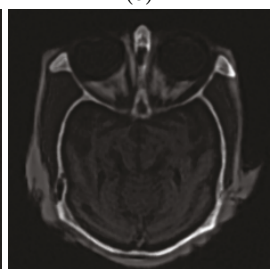

(y)

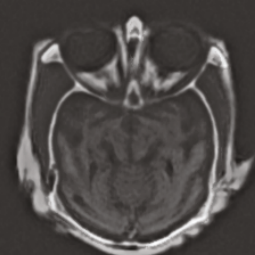

(c)

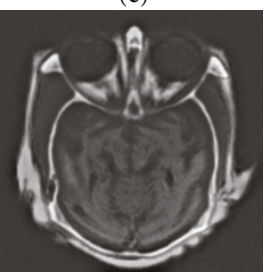

(h)

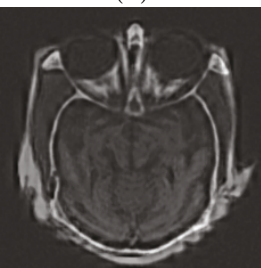

(n)

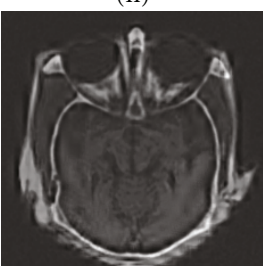

(t)

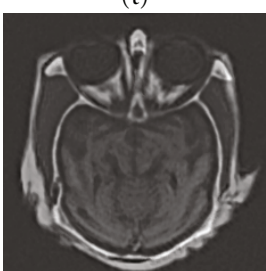

(z)

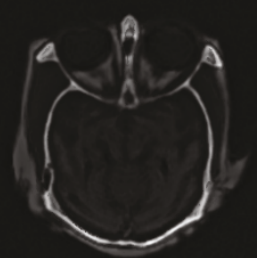

(d)

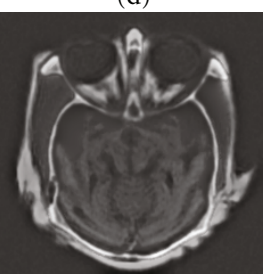

(i)

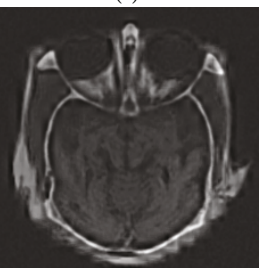

(o)

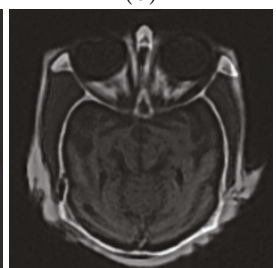

(u)

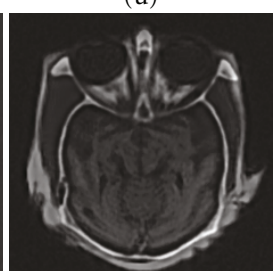

(aa)

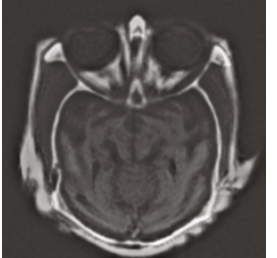

(e)

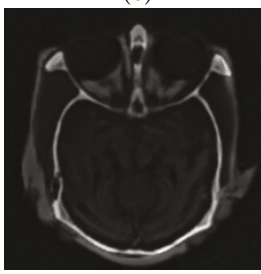

(j)

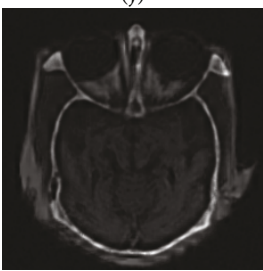

(p)

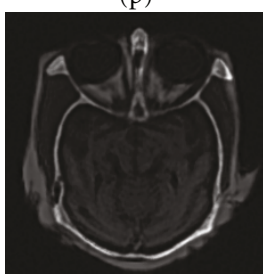

(v)

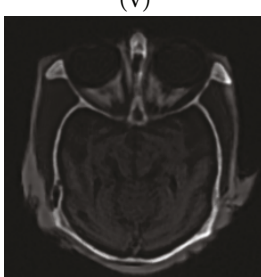

(bb)

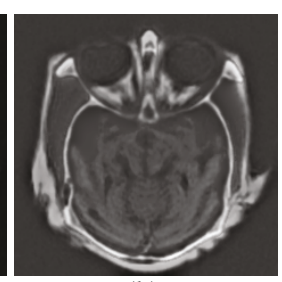

(k)

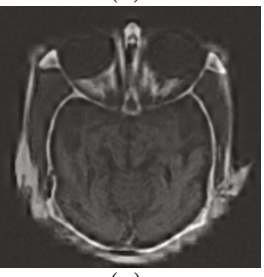

(q)

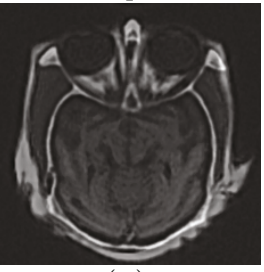

(w)

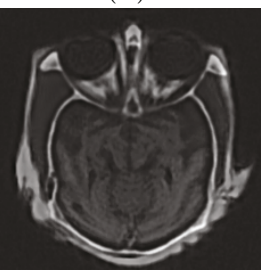

(cc)

Figure 11: (a) and (b) Original "brain" source images, image fusion results using (c) LP/AM, (d) LIP-LP/AM, (e) PL-LP/AM, (f) LP/BK, (g) LIP-LP/BK, (h) PL-LP/BK, (i) LP/RB, (j) LIP-LP/RB, (k) PL-LP/RB, (l) DWT/AM, (m) LIP-DWT/AM, (n) PL-DWT/AM, (o) DWT/BK, (p) LIP-DWT/BK, (q) PL-DWT/BK, (r) DWT/RB, (s) LIP-DWT/RB, (t) PL-DWT/RB, (u) SWT/AM, (v) LIP-SWT/AM, (w) PL-SWT/AM, (x) SWT/BK, (y) LIP-SWT/BK, (z) PL-SWT /BK, (aa) SWT/RB, (bb) LIP-SWT/RB, (cc) PL-SWT/RB.

regions in which the saliency of the source images is greater. Defining the overall saliency of a window $C(w)$ by

$$
C(w)=\max \left(s\left(I_{1} \mid w\right), s\left(I_{2} \mid w\right)\right) .
$$

The weighted fusion quality index $Q_{W}\left(I_{1}, I_{2}, F\right)[25]$ is given by

$$
\begin{aligned}
& Q_{w}\left(I_{1}, I_{2}, F\right) \\
& =\sum_{w \in W} c(w)\left(\lambda(w) Q_{0}\left(I_{1}, F \mid w\right)+(1-\lambda(w)) Q_{0}\left(I_{2}, F \mid w\right)\right),
\end{aligned}
$$

where

$$
c(w)=\frac{C(w)}{\sum_{w^{\prime} \in W} C\left(w^{\prime}\right)}
$$

As $Q_{0}$ yields a maximum value of 1 for identical input images, higher fusion quality metric values indicate better fusion results. Figure 8 provides a graphical representation of the weights which are calculated by the quality metric in order to assess the quality of image fusion results.

\section{Experimental Results}

The effectiveness of the proposed algorithms is illustrated via computer simulations. In general, three cases are considered 


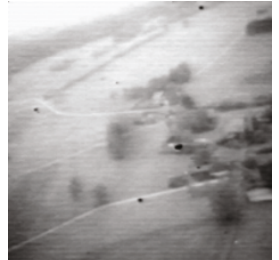

(a)

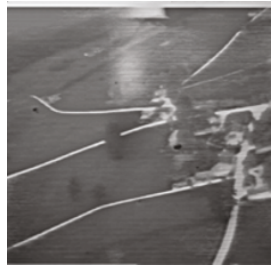

(f)

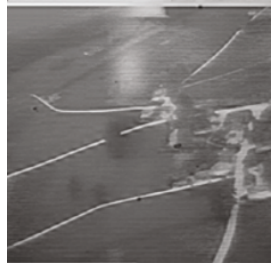

(1)

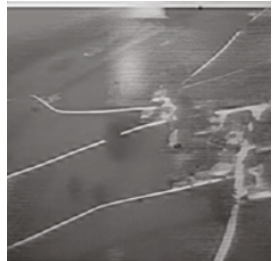

$(\mathrm{r})$

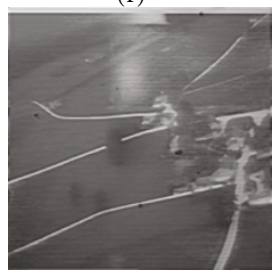

$(\mathrm{x})$

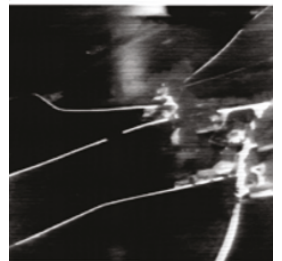

(b)

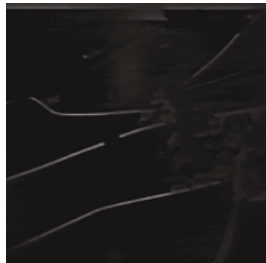

(g)

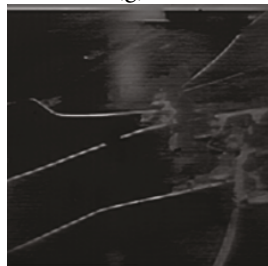

(m)

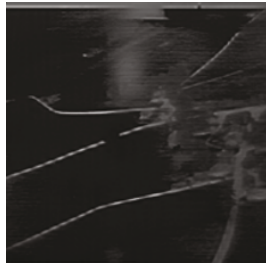

$(\mathrm{s})$

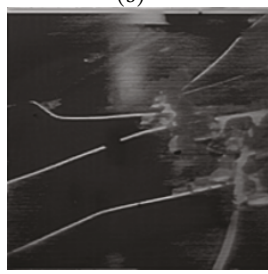

(y)

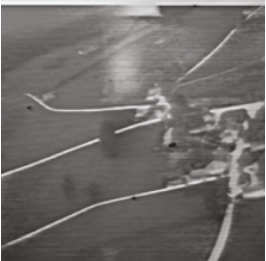

(c)

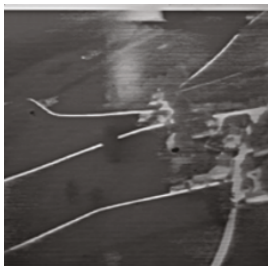

(h)

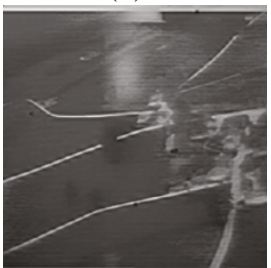

(n)

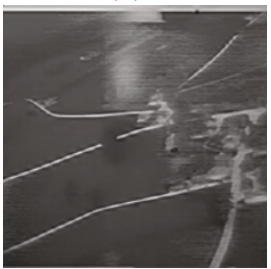

(t)

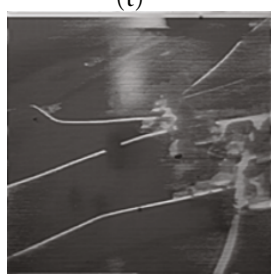

(z)

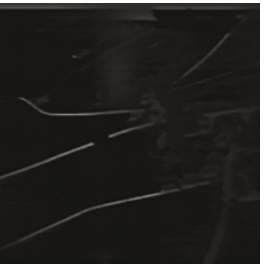

(d)

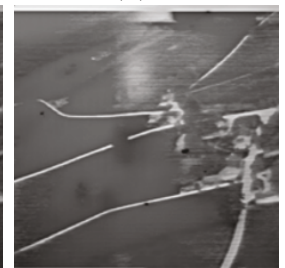

(i)

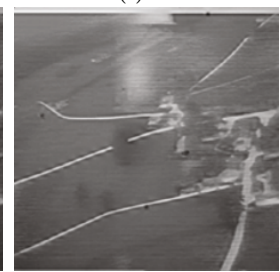

(o)

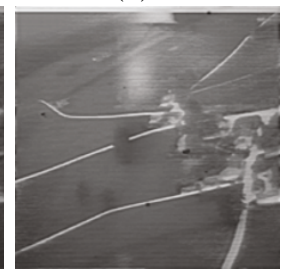

(u)

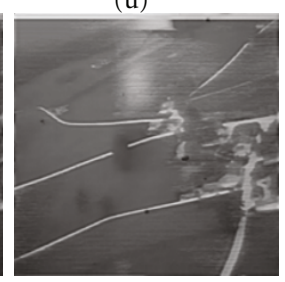

(aa)

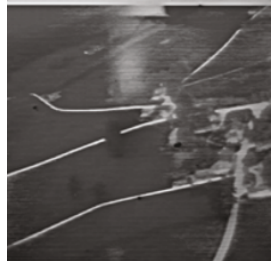

(e)

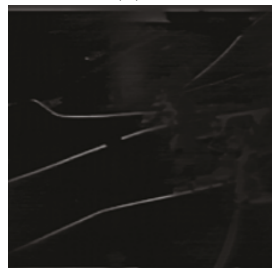

(j)

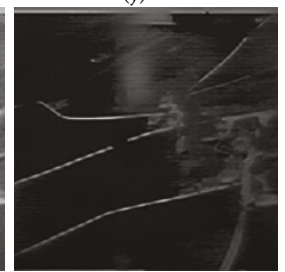

(p)

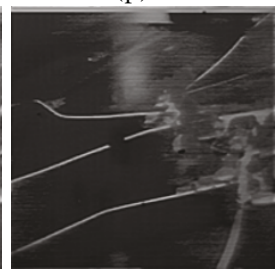

(v)

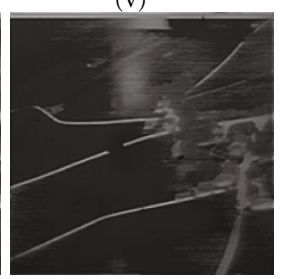

(bb)

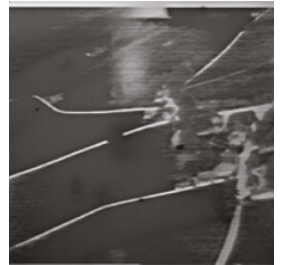

(k)

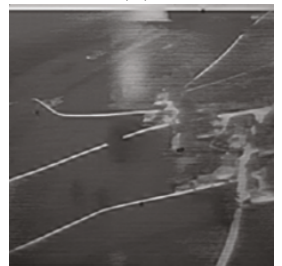

(q)

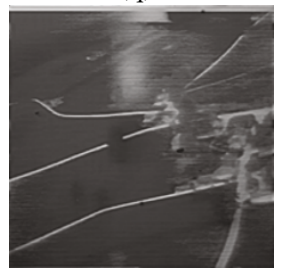

$(\mathrm{w})$

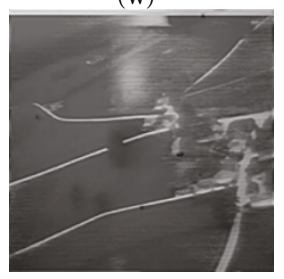

(cc)

FIGURE 12: (a) and (b) Original "navigation" source images, image fusion results using (c) LP/AM, (d) LIP-LP/AM, (e) PL-LP/AM, (f) LP/BK, (g) LIP-LP/BK, (h) PL-LP/BK, (i) LP/RB, (j) LIP-LP/RB, (k) PL-LP/RB, (l) DWT/AM, (m) LIP-DWT/AM, (n) PL-DWT/AM, (o) DWT/BK, (p) LIP-DWT/BK, (q) PL-DWT/BK, (r) DWT/RB, (s) LIP-DWT/RB, (t) PL-DWT/RB, (u) SWT/AM, (v) LIP-SWT/AM, (w) PL-SWT/AM, (x) SWT/BK, (y) LIP-SWT/BK, (z) PL-SWT /BK, (aa) SWT/RB, (bb) LIP-SWT/RB, (cc) PL-SWT/RB.

for these experiments: (1) the extreme case in which the PLIP model operators yield the LIP model operators $(\gamma=M)$, (2) standard operators, which are the extreme case of PLIP model operators with $\gamma=\infty$, (3) the case in which $\gamma$ takes on a value other than $M$ or $\infty$. For easy reference, we refer to these cases as the LIP model operator case, standard operator case, and PLIP model operator case, respectively, though in reality, all are cases of the proposed PLIP-based approach. It should be noted that image fusion algorithms employing LIP-based multiresolution image decomposition schemes and fusion rules have not even been introduced to our knowledge. Thus, we refer to the LIP-LP, LIP-DWT, and LIPSWT image fusion algorithms as the image fusion algorithms which use PLIP operators with $\gamma=M$ to implement the fusion rules and LP, DWT, and SWT, respectively.
Consequently, the PL-LP, PL-DWT, and PL-SWT image fusion algorithms are compared to the traditional LP and LIP-LP; traditional DWT and LIP-DWT; and traditional and LIP SWT image fusion algorithms, respectively. The algorithms were tested over a range of different image classes, including out-of-focus, medical, surveillance, and remote sensing images. A portion of these results are presented here. It is assumed that the input source images are registered, although it is expected that image fusion algorithms be able to handle minor registration differences. There are many factors which influence image fusion using multiresolution decomposition schemes, including the type of multiresolution decomposition scheme, the number of decomposition levels, the choice of filter bank, and the fusion rule used to fuse coefficients at each scale. This paper 


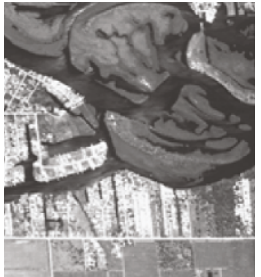

(a)

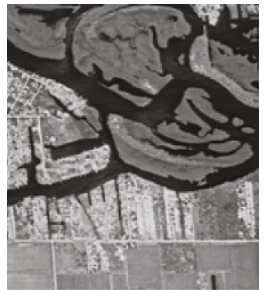

(f)

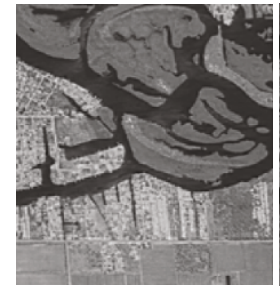

(1)

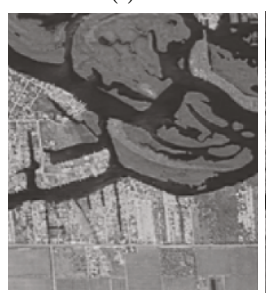

(r)

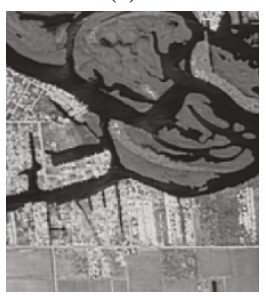

(x)

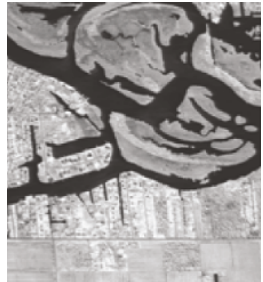

(b)

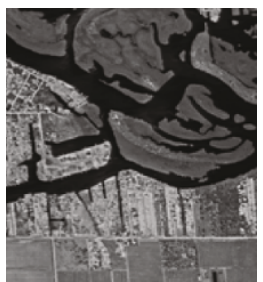

(g)

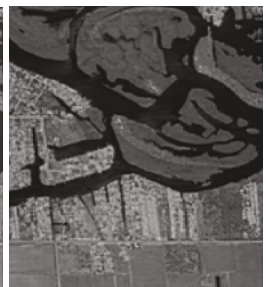

(m)

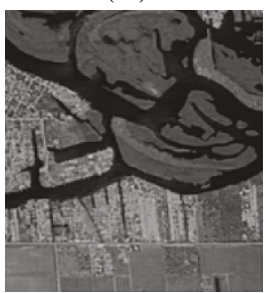

(s)

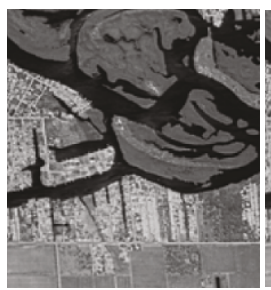

(y)

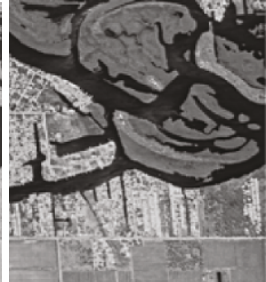

(c)

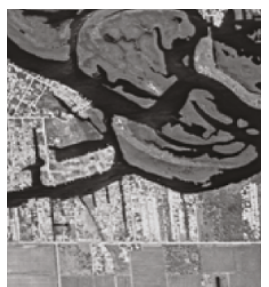

(h)

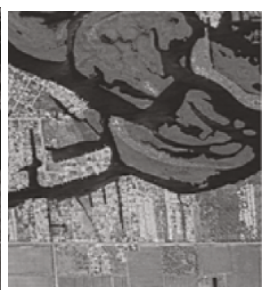

(n)

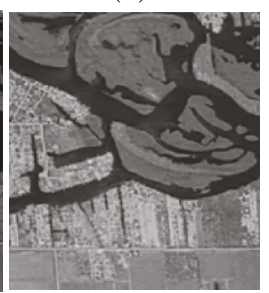

(t)

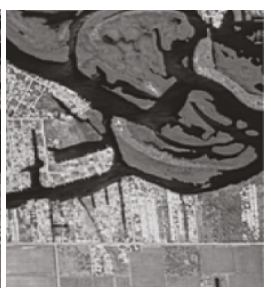

( $\mathrm{z})$

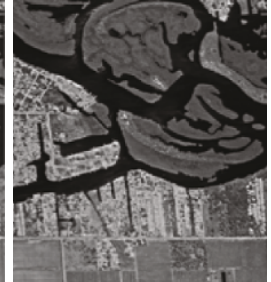

(d)

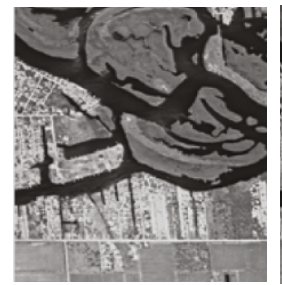

(i)

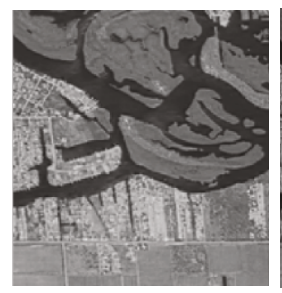

(o)

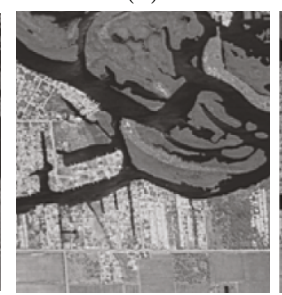

(u)

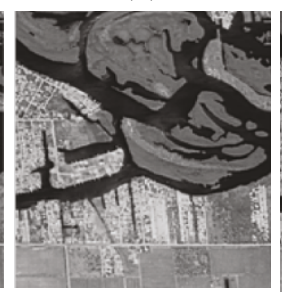

(aa)

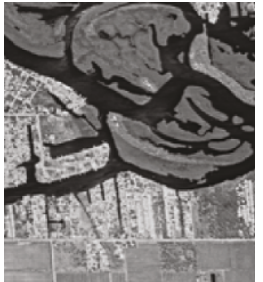

(e)

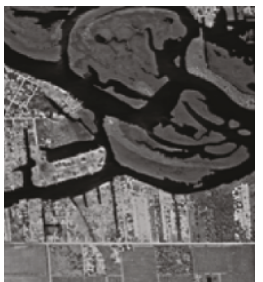

(j)

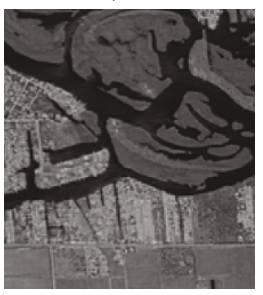

(p)

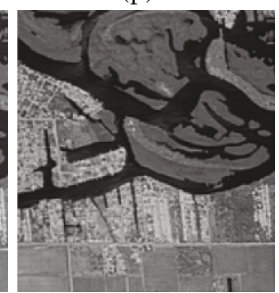

(v)

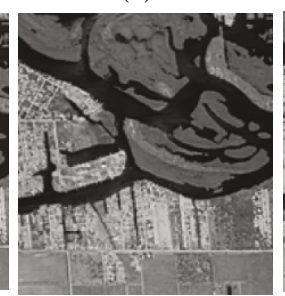

(bb)

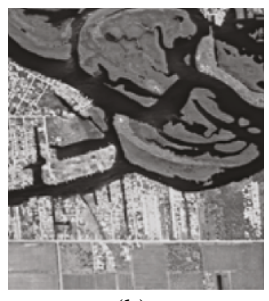

(k)

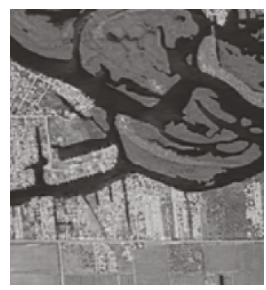

(q)

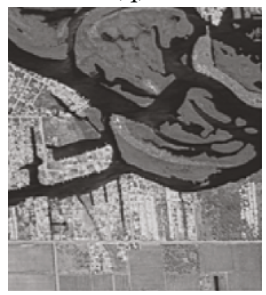

(w)

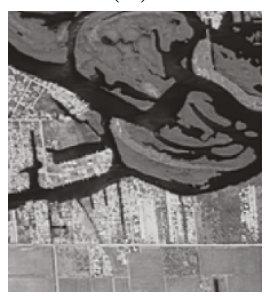

(cc)

FIgURE 13: (a) and (b) Original "remote sensing" source images, image fusion results using (c) LP/AM, (d) LIP-LP/AM, (e) PL-LP/AM, (f) LP/BK, (g) LIP-LP/BK, (h) PL-LP/BK, (i) LP/RB, (j) LIP-LP/RB, (k) PL-LP/RB, (l) DWT/AM, (m) LIP-DWT/AM, (n) PL- DWT/AM, (o) DWT/BK, (p) LIP-DWT/BK, (q) PL-DWT/BK, (r) DWT/RB, (s) LIP-DWT/RB, (t) PL-DWT/RB, (u) SWT/AM, (v) LIP-SWT/AM, (w) PL-SWT/AM, (x) SWT/BK, (y) LIP-SWT/BK, (z) PL-SWT /BK, (aa) SWT/RB, (bb) LIP-SWT/RB, (cc) PL-SWT/RB.

emphasizes the transform which is used while keeping all other factors constant. In these experimental results, $N=$ 3 for all methods, and both the pixel- and region-based fusion rules are examined. For the wavelet-based approaches, biorthogonal 2.2 filters are used. The fusion results are compared quantitatively by first normalizing source images and fused results to the range $0-255$, and then using the Piella and Heijmans image fusion quality metric $Q_{W}$ with $w=7$. This metric is used to determine the optimal parameter value for $\gamma$, with the resulting fused image thereby taken to be the result for a given parameterized logarithmic image fusion algorithm. This demonstrates the ability to tune the PLIP model parameter in order to optimize results according to any metric used for quality assessment.

Zoomed details highlighting specific contrast differences of selected fusion results are shown in Figure 9. Complete image fusion results showing more global luminance differences can be found in Figures 10, 11, 12, and 13. Qualitatively, it is seen that the image fusion approaches using the PLIP model operator case yield more informative fusion results with more visually pleasing contrast. The zoomed details in the 1st row of Figure 9 show that the lines and numbers in the clock images are sharper and clearer in the fusion result using the PLIP model operator case. 
TABLe 2: Quantitative quality assessment of image fusion results using the Piella and Heijmans quality metric.

\begin{tabular}{|c|c|c|c|c|c|c|c|c|c|c|c|c|c|}
\hline \multirow{2}{*}{$\begin{array}{l}\text { Decomposition } \\
\text { scheme }\end{array}$} & \multirow{2}{*}{$\begin{array}{l}\text { Fusion } \\
\text { rule }\end{array}$} & \multicolumn{3}{|c|}{ Clocks } & \multicolumn{3}{|c|}{ Brain } & \multicolumn{3}{|c|}{ Navigation } & \multicolumn{3}{|c|}{ Remote sensing } \\
\hline & & Standard & LIP & PLIP & Standard & LIP & PLIP & Standard & LIP & PLIP & Standard & LIP & PLIP \\
\hline \multirow{3}{*}{ LP } & $\mathrm{AM}$ & 0.8914 & 0.9168 & 0.9300 & 0.7753 & 0.5256 & 0.7760 & 0.7947 & 0.3467 & 0.8200 & 0.8383 & 0.7842 & 0.8404 \\
\hline & $\mathrm{BK}$ & 0.8851 & 0.9123 & 0.9250 & 0.7748 & 0.5349 & 0.7762 & 0.7933 & 0.3512 & 0.8196 & 0.8293 & 0.7627 & 0.8300 \\
\hline & $\mathrm{RB}$ & 0.8849 & 0.9114 & 0.9241 & 0.7572 & 0.5327 & 0.7576 & 0.8051 & 0.3505 & 0.8187 & 0.8113 & 0.7424 & 0.8120 \\
\hline \multirow{3}{*}{ DWT } & $\mathrm{AM}$ & 0.8750 & 0.8979 & 0.9002 & 0.7124 & 0.5296 & 0.7292 & 0.7363 & 0.6011 & 0.7607 & 0.7672 & 0.7128 & 0.7695 \\
\hline & $\mathrm{BK}$ & 0.8745 & 0.8891 & 0.8918 & 0.6701 & 0.4886 & 0.6886 & 0.7333 & 0.6064 & 0.7600 & 0.7378 & 0.6770 & 0.7385 \\
\hline & $\mathrm{RB}$ & 0.8763 & 0.8955 & 0.8972 & 0.6872 & 0.5008 & 0.7060 & 0.7288 & 0.6052 & 0.7589 & 0.7162 & 0.6869 & 0.7170 \\
\hline \multirow{3}{*}{ SWT } & AM & 0.8879 & 0.9085 & 0.9134 & 0.7539 & 0.5581 & 0.7718 & 0.7460 & 0.7250 & 0.7746 & 0.8137 & 0.7954 & 0.8150 \\
\hline & $\mathrm{BK}$ & 0.8926 & 0.9081 & 0.9130 & 0.7554 & 0.5714 & 0.7647 & 0.7382 & 0.7294 & 0.7821 & 0.8203 & 0.8045 & 0.8238 \\
\hline & $\mathrm{RB}$ & 0.8877 & 0.9045 & 0.9064 & 0.7458 & 0.5557 & 0.7684 & 0.7542 & 0.6873 & 0.7695 & 0.8078 & 0.7882 & 0.8080 \\
\hline
\end{tabular}

The 2nd row shows that the proposed method is able to better capture the terrain information and road information of the respective source images. The 3 rd row shows the improved contrast of tissue information and dense bone structure yielded by the proposed method. Lastly, the 4th row shows that the proposed fusion approaches are able to better capture the subtle features at the point at which the roads intersect. Thus, the experimental results highlight the improvement of fusion results yielded using the PLIP model operators. While the standard operator extreme can often give adequate results, the contrast and luminance can be improved by choosing a value of $\gamma$ which both reflects the human visual system and meets the dynamic range requirements of the input images. While the LIP model operator extreme can improve the performance of image fusion relative to standard operator extreme when the source images are similar in luminance (as in the case of the clocks images), it yields visually inadequate results for source images with greatly different local base luminance. This is particularly visible for input images in which one of the source images is predominantly dark as in the case of the "navigation" and "brain" images.

The quantitative observations are reflected by their corresponding quality metric values in Table 2 , in which rows correspond to the basic multiresolution decomposition scheme and fusion rule employed and columns correspond to the image processing operators (LIP model operator case, standard operator case, or PLIP model operator case) used to implement the given decomposition scheme and fusion rule. It should be noted that a single, constant-size window is used in calculating the quality metric values. Thus, such an evaluation may be dependent on how well the window size reflects the scale of the objects of interest in the source images and may not be able to effectively quantify differences in fusion results even when qualitative visual differences are seen. This provides a rationalization as to why the perceived visual improvement of the proposed methods may in some cases only translate to a small increase in the quality metric values and continues to affirm the fact that objective image fusion quality assessment is still an open research topic. However, the rank of the scores is generally indicative of relative performance, and to standardize the testing procedure and to maintain the same formulation of the metric as it was originally proposed, the same parameters are used to calculate quality metric values for all test cases. Thus, the quantitative analysis serves as an objective means of validating subjective observations. The quality metric values in Table 2 show that, in all cases, fusion algorithms using the parameterized logarithmic multiresolution decomposition schemes and fusion rules outperform their respective general linear processing model counterparts.

\section{Conclusions}

This paper derived decomposition schemes and image fusion rules based on the PLIP model. The PLIP-based multiresolution decomposition schemes were developed and thoroughly applied for image fusion purposes. PLIP model properties were analyzed, and their implications for image fusion were verified by experimental means. The new multiresolution decomposition schemes and fusion rules yield new image fusion tools which are able to provide visually more pleasing fusion results. A new class of image fusion algorithms, namely, those based on the PL-LP, PL-DWT, and PL-SWT were proposed. The images are fused in the transform domain using novel pixel-based or region-based rules. Using a number of pixel-based and region-based fusion rules, one can combine the important features of the input images in the transform domain to compose an enhanced image. The proposed algorithms were tested and compared to traditional and LIP multiresolution image fusion algorithms over a number of different image classes including outof-focus, medical, surveillance, and remote sensing images, whose applications can make use of image fusion to improve perception for computer-aided or computer vision systems. These experimental results showed that the proposed image decomposition and image algorithms improved image fusion quality both qualitatively and quantitatively. Qualitatively, the fusion results using the proposed algorithms provided better contrast and the necessary luminance needed for fusion purposes. Quantitatively, the proposed algorithms outperformed traditional and LIP multiresolution image fusion algorithms using the Piella and Heijmans quality metric. 


\section{Acknowledgments}

This work has been partially supported by NSF Grant HRD-0932339. The authors would like to thank Dr. Oliver Rockinger for kindly providing the registered images used for computer simulations and to the anonymous referees for their invaluable suggestions which substantially improved the quality of this paper.

\section{References}

[1] G. Piella, "A general framework for multiresolution image fusion: from pixels to regions," Information Fusion, vol. 4, no. 4, pp. 259-280, 2003.

[2] P. Hill, N. Canagarajah, and D. Bull, "Image fusion using complex wavelets," in Proceedings of the 13th British Machine Vision Conference, pp. 487-496, Cardiff, UK, 2002.

[3] M. Kumar and S. Dass, "A total variation-based algorithm for pixel-level image fusion," IEEE Transactions on Image Processing, vol. 18, no. 9, pp. 2137-2143, 2009.

[4] S. Daneshvar and H. Ghassemian, "MRI and PET image fusion by combining IHS and retina-inspired models," Information Fusion, vol. 11, no. 2, pp. 114-123, 2010.

[5] Y. Yang, D. S. Park, S. Huang, and N. Rao, "Medical image fusion via an effective wavelet-based approach," EURASIP Journal on Advances in Signal Processing, vol. 2010, Article ID 579341, 13 pages, 2010.

[6] Z. Zhang and R. Blum, "Region-based image fusion scheme for concealed weapon detection," in Proceedings of the 31st Annual Conference on Information Sciences and Systems, pp. 168-173, 1997.

[7] X.-Q. Zhang, Z.-S. Gao, and H.-Z. Yuan, "Dynamic infrared and visible image sequence fusion based on DT-CWT using GGD," in Proceedings of the International Conference on Computer Science and Information Technology (ICCSIT '08), pp. 875-878, September 2008.

[8] Y. Chibani, "Selective synthetic aperture radar and panchromatic image fusion by using the à trous wavelet decomposition," EURASIP Journal on Applied Signal Processing, vol. 2005, no. 14, pp. 2207-2214, 2005.

[9] S.-Y. Zhang, P. Wang, X. Chen, and X. Zhang, "A new method for multi-source remote sensing image fusion," in Proceedings of the IEEE International Geoscience and Remote Sensing Symposium (IGARSS '05), pp. 3948-3951, July 2005.

[10] Z. Zhang and R. S. Blum, "A categorization of multiscaledecomposition-based image fusion schemes with a performance study for a digital camera application," Proceedings of the IEEE, vol. 87, no. 8, pp. 1315-1326, 1999.

[11] P. S. Chavez Jr. and A. Y. Kwarteng, "Extracting spectral contrast in Landsat Thematic Mapper image data using selective principal component analysis," Photogrammetric Engineering \& Remote Sensing, vol. 55, no. 3, pp. 339-348, 1989.

[12] T.-M. Tu, S.-C. Su, H.-C. Shyu, and P. S. Huang, "Efficient intensity-hue-saturation-based image fusion with saturation compensation," Optical Engineering, vol. 40, no. 5, pp. 720 728, 2001.

[13] M. Tabb and N. Ahuja, "Multiscale image segmentation by integrated edge and region detection," IEEE Transactions on Image Processing, vol. 6, no. 5, pp. 642-655, 1997.

[14] P. J. Burt and E. H. Adelson, "Lapacian pyramid as a compact image code," IEEE Transactions on Communications, vol. 31, no. 4, pp. 532-540, 1983.
[15] S. G. Mallat, "Theory for multiresolution signal decomposition: the wavelet representation," IEEE Transactions on Pattern Analysis and Machine Intelligence, vol. 11, no. 7, pp. 674-693, 1989.

[16] O. Rockinger, "Image sequence fusion using a shift-invariant wavelet transform," in Proceedings of the International Conference on Image Processing, pp. 288-291, October 1997.

[17] M. Jourlin and J. C. Pinoli, "Logarithmic image processing: the mathematical and physical framework for the representation and processing of transmitted images," Advances in Imaging and Electron Physics, vol. 115, pp. 126-196, 2001.

[18] J.-C. Pinoli, "A general comparative study of the multiplicative homomorphic, log-ratio and logarithmic image processing approaches," Signal Processing, vol. 58, no. 1, pp. 11-45, 1997.

[19] G. Deng, L. W. Cahill, and G. R. Tobin, "The study of logarithmic image processing model and its application to image enhancement," IEEE Transaction on Image Processing, vol. 18, pp. 1135-1140, 2009.

[20] C. Vertan, A. Oprea, C. Florea, and L. Florea, "A pseudologarithmic image processing framework for edge detection," in Advanced Concepts for Intelligent Vision Systems, vol. 5259 of Lecture Notes in Computer Science, pp. 637-644, 2008.

[21] J. Debayle, Y. Gavet, and J.-C. Pinoli, "General adaptive neighborhood image restoration, enhancement and segmentation," in Proccedings of the International Conference on Image Analysis and Recognition (ICIAR '06), vol. 4141 of Lecture Notes in Computer Science, pp. 29-40, Springer, Heidelberg, Germany, 2006.

[22] K. A. Panetta, E. J. Wharton, and S. S. Agaian, "Human visual system-based image enhancement and logarithmic contrast measure," IEEE Transactions on Systems, Man, and Cybernetics, Part B, vol. 38, no. 1, pp. 174-188, 2008.

[23] E. J. Wharton, K. Panetta, and S. S. Agaian, "Logarithmic edge detection with applications," in IEEE International Conference on Systems, Man, and Cybernetics (SMC '07), pp. 3346-3351, October 2007.

[24] E. Wharton, S. Agaian, and K. Panetta, "Comparative study of logarithmic enhancement algorithms with performance measure," in Image Processing: Algorithms and Systems, Neural Networks, and Machine Learning, vol. 6064 of Proceedings of the SPIE, p. 342353, San Jose, Calif, USA, 2006.

[25] G. Piella and H. Heijmans, "A new quality metric for image fusion," in Proceedings of International Conference on Image Processing (ICIP '03), vol. 2, pp. 173-176, September 2003.

[26] J. M. Palomares, J. Gonzalez, and E. Ros, "Designing a fast convolution under the LIP paradigm applied to edge detection," in Proceedings of the 3rd International Conference on Advances in Pattern Recognition (ICAPR '05), vol. 3687 of Lecture Notes in Computer Science, pp. 560-569, Bath, UK, August 2005.

[27] J. E. Fowler, "The redundant discrete wavelet transform and additive noise," IEEE Signal Processing Letters, vol. 12, no. 9, pp. 629-632, 2005.

[28] G. Courbebaisse, F. Trunde, and M. Journlin, "Wavelet transform and LIP model," Image Analysis and Stereology, vol. 21, no. 2, pp. 121-125, 2002.

[29] P. J. Burt and R. J. Kolczynski, "Enhanced image capture through fusion," in Proceedings of the 4th International Conference on Computer Vision, pp. 173-182, May 1993.

[30] J. J. Lewis, R. J. O'Callaghan, S. G. Nikolov, D. R. Bull, and C. N. Canagarajah, "Region-based image fusion using complex wavelets," in Proceedings of the Seventh International Conference on Information Fusion (FUSION '04), pp. 555-562, July 2004. 
[31] A. M. Khan, B. Kayani, and A. M. Gillani, "Feature level fusion of night vision images based on K-means clustering algorithm," in Innovations and Advanced Techniques in Computer and Information Sciences and Engineering, pp. 73-76, Springer, 2007.

[32] Z. Li, Z. Jing, G. Liu, S. Sun, and H. Leung, "A region-based image fusion algorithm using multiresolution segmentation," in Proceedings of the IEEE International Conference on Intelligent Transportation Systems, vol. 1, pp. 96-101, 2003.

[33] L. Shuang and L. Zhilin, "A region-based technique for fusion of high-resolutino images using mean shift segmentation," in International Archives of the Photogrammetry, Remote Sensing, and Spatial Information Sciences, vol. 38, pp. 1267-1272, Beijing, China, 2008.

[34] D. Comaniciu and P. Meer, "Mean shift: a robust approach toward feature space analysis," IEEE Transactions on Pattern Analysis and Machine Intelligence, vol. 24, no. 5, pp. 603-619, 2002.

[35] W. Tao, H. Jin, and Y. Zhang, "Color image segmentation based on mean shift and normalized cuts," IEEE Transactions on Systems, Man, and Cybernetics, Part B, vol. 37, no. 5, pp. 1382-1389, 2007.

[36] C. S. Xydeas and V. Petrović, "Objective image fusion performance measure," Electronics Letters, vol. 36, no. 4, pp. 308-309, 2000.

[37] G. Qu, D. Zhang, and P. Yan, "Information measure for performance of image fusion," Electronics Letters, vol. 38, no. 7, pp. 313-315, 2002.

[38] Z. Wang and A. C. Bovik, "A universal image quality index," IEEE Signal Processing Letters, vol. 9, no. 3, pp. 81-84, 2002. 